\title{
DECAY PROPERTY OF STOPPED MARKOVIAN BULK-ARRIVING QUEUES
}

\author{
JUNPING LI, ${ }^{*}$ Central South University \\ ANYUE CHEN, ${ }^{* *}$ University of Liverpool and University of Hong Kong
}

\begin{abstract}
We consider decay properties including the decay parameter, invariant measures, invariant vectors, and quasistationary distributions of a Markovian bulk-arriving queue that stops immediately after hitting the zero state. Investigating such behavior is crucial in realizing the busy period and some other related properties of Markovian bulk-arriving queues. The exact value of the decay parameter $\lambda_{C}$ is obtained and expressed explicitly. The invariant measures, invariant vectors, and quasistationary distributions are then presented. We show that there exists a family of invariant measures indexed by $\lambda \in\left[0, \lambda_{C}\right]$. We then show that, under some conditions, there exists a family of quasistationary distributions, also indexed by $\lambda \in\left[0, \lambda_{C}\right]$. The generating functions of these invariant measures and quasistationary distributions are presented. We further show that a stopped Markovian bulk-arriving queue is always $\lambda_{C}$-transient and some deep properties are revealed. The clear geometric interpretation of the decay parameter is explained. A few examples are then provided to illustrate the results obtained in this paper.
\end{abstract}

Keywords: Markovian bulk-arriving queue; decay parameter; invariant measure; invariant vector; quasistationary distribution

2000 Mathematics Subject Classification: Primary 60J27

Secondary $60 \mathrm{~J} 35$

\section{Introduction}

Markovian queues are the most basic yet possibly the most important branch of applied probability. On the one hand, they interweave the general theory of queueing models, while, on the other hand, they interweave the general theory and applications of continuous-time Markov chains, and have become a very successful and fruitful research field. Good references, among many others, are [2] [17], [24], and [27] for the former and [1], [10], [32], and [38], for the latter. See also [8] and [9], which contain new information about continuous-time Markov chains. Within this queueing framework, the bulk-arriving queues occupy a major niche and play an important role both in the theory and applications of Markovian queueing models, and have attracted considerable attention, mainly owing to their extensive applications in many practical situations experienced in science and technology such as in industrial assembly lines, road traffic flow, arrival of aircraft passengers, etc. An excellent reference for bulk queues is [4]. This field has close theoretical links with the versatile Markovian point process addressed in Neuts [29], in which many types of bulk-arrival processes were examined. In addition, Neuts [30] provided many interesting bulk-arriving models together with useful methods and techniques for their

Received 8 June 2007; revision received 28 November 2007.

* Postal address: School of Mathematical Science and Computing Technology, Central South University, Changsha, 410075, P. R. China. Email address: jpli@mail.csu.edu.cn

** Postal address: Division of Statistics and Probability, Department of Mathematical Sciences, The University of Liverpool, Liverpool, L69 7ZL, UK. Email address: achen@liv.ac.uk 
analysis. For further discussions on Markovian bulk-arriving queues, see [13], [25], [26], [31], and [37].

Markovian queueing models with state-independent and state-dependent input/output have also attracted considerable research interest. For example, Parthasarthy and Krishna [33] allowed arbitrary input when the queue was empty. Chen and Renshaw [5], [6] introduced the possibility of removing the entire workload. Another new concept in queueing theory is the so-called negative arrivals. It seems that Gelenbe [15] and Gelenbe et al. [16] first introduced this particularly interesting concept, whilst other related papers include [3], [18], [19], and [20].

Because of this extensive interest, many deep properties regarding Markovian bulk-arriving queues, such as recurrence and positive recurrence criteria, the queueing length and the busy period distributions, and the long-run behavior under the positive-recurrent scenario have been revealed. See the references mentioned above.

However, it seems that there exists an important question which has not been extensively addressed. That is, what is the long-run behavior, at least in some sense, if the Markovian queue concerned is transient? Another closely related but perhaps more important question is, what does the long-run behavior look like for the busy period for both the transient and recurrent Markovian bulk queues? Apparently, a deep understanding of such conditional longrun behavior would be extremely helpful in analyzing and designing complex queueing systems.

In fact, with the development of the general theory and applications of continuous-time Markov chains, there has been a long history of investigating several closely linked and very important concepts regarding the conditional long-run behavior, i.e. the important concepts of decay parameter, invariant measures, and quasistationary distributions. The idea of using quasistationary distributions can be traced back at least to the early work of Yaglom [43], who considered the long-run behavior, in a sense which will be explained later, of the subcritical Galton-Watson process. The other important concept, the decay parameter, was developed by Kingman in the early 1960s. Beginning with the pioneering and remarkable work of Kingman [23] and Vere-Jones [42], this extremely useful theory has flourished owing to much important research, including the significant contributions made by [12], [14], [21], [22], [28], [34], [35], [36], [39], [40], [41], and many others.

Roughly speaking, invariant measures and quasistationary distributions can be used to model the long-term behavior of many stochastic systems in a variety of diverse contexts in which the systems will stop moving, but appear to be stationary, in some sense, over any reasonable time scale. For example, in the so-called stopped Markovian queue, which will be the main topic of this paper, the queueing system may be empty at some time epoch, and then the busy period ends. Thus, it is important and interesting to find the long-term behavior of such queueing systems under the condition that the queueing system has not hit the zero state yet. Other examples can be found in chemical reaction kinetics, population models, etc.

The important point is that Kingman [23] showed that, for an irreducible class $C$ of any continuous-time Markov chain, there exists a number $\lambda_{C} \geq 0$, called the decay parameter of the corresponding process, such that, for all $i, j \in C$,

$$
\frac{1}{t} \log p_{i j}(t) \rightarrow-\lambda_{C} \quad \text { as } t \rightarrow+\infty,
$$

from which we may clearly see that the decay parameter represents some kind of convergent rate regarding the long-run behavior. The other way to characterize this important quantity is as follows. Let

$$
\mu_{i j}=\inf \left\{\lambda \geq 0: \int_{0}^{\infty} \mathrm{e}^{\lambda t} p_{i j}(t) \mathrm{d} t=\infty\right\}=\sup \left\{\lambda \geq 0: \int_{0}^{\infty} \mathrm{e}^{\lambda t} p_{i j}(t) \mathrm{d} t<\infty\right\} .
$$


Then, by the irreducibility argument, it is fairly easy to show that $\mu_{i j}$ does not depend on $i, j \in C$. Denote this common value of $\mu_{i j}$ by $\mu$. It is straightforward to show that the common abscissa of convergence of these integrals is just the decay parameter, i.e.

$$
\lambda_{C}=\mu \text {. }
$$

The decay parameter and the quasistationary distributions are closely linked to the socalled $\mu$-subinvariant/invariant measures and $\mu$-subinvariant/invariant vectors. An elementary but detailed discussion of this theory, including the basic definitions involved, can be found in Chapter 5 of [1]. For convenience, we now repeat the definition of the quasistationary distribution.

Definition 1.1. Suppose that $\left(p_{i j}(t) ; i, j \in \mathbb{E}\right)$ is a transition function of a continuous-time Markov chain defined on the state space $\mathbb{E}$. Assume that $C$ is a communicating class of $\mathbb{E}$ and that $\left(m_{i} ; i \in C\right)$ is a probability distribution over $C$. Let

$$
p_{j}(t)=\sum_{i \in C} m_{i} p_{i j}(t) \quad \text { for } j \in C \text { and } t \geq 0
$$

If

$$
\frac{p_{j}(t)}{\sum_{i \in C} p_{i}(t)}=m_{j}, \quad j \in C, t>0,
$$

then $\left(m_{i} ; i \in C\right)$ is called a quasistationary distribution for $\left(p_{i j}(t) ; i, j \in \mathbb{E}\right)$.

The deep relationships between the decay parameter, the invariant measures, and the quasistationary distributions have been revealed in the important works of Nair and Pollett [28] and Van Doorn [41].

The main aim of this paper is to extensively and comprehensively investigate the decay properties of a Markovian bulk-arriving queue which stops after hitting the zero state. This model is usually called the stopped Markovian bulk-arriving queue (or the stopped $M^{X} / M / 1$ queue). Investigating such properties is crucial in realizing the behavior of the busy period of the corresponding queueing systems. It will also be the key step in investigating the conditional long-run behavior under the transient scenario of the Markovian bulk-arriving queues with or without state-dependent input/output. The generator matrix, i.e. the so-called $q$-matrix $\boldsymbol{Q}=\left(q_{i j} ; i, j \in \mathbb{Z}_{+}\right)$, where $\mathbb{Z}_{+}$stands for the nonnegative integers $\{0,1,2, \ldots\}$, of such queueing models is given as follows:

$$
q_{i j}= \begin{cases}b_{j-i+1} & \text { if } i \geq 1, j \geq i-1, \\ 0 & \text { otherwise }\end{cases}
$$

where

$$
b_{j} \geq 0, \quad j \neq 1, \quad 0<\sum_{j \neq 1} b_{j} \leq-b_{1}<\infty .
$$

In order to avoid discussing some trivial cases, we shall assume throughout this paper that $b_{0}>0$ and $\sum_{j=2}^{\infty} b_{j}>0$. An immediate consequence of these assumptions is that $C=\{1,2, \ldots\}$ is an irreducible class for $\boldsymbol{Q}$ as well as for the corresponding $\boldsymbol{Q}$-process. The latter is formally defined as follows.

Definition 1.2. Let $\boldsymbol{Q}=\left(q_{i j} ; i, j \in \mathbb{Z}_{+}\right)$be the generator matrix defined in (1.1)-(1.2). The corresponding transition function $\boldsymbol{P}(t)=\left(p_{i j}(t) ; i, j \in \mathbb{Z}_{+}\right)$is called the stopped Markovian bulk-arriving queueing process (or stopped $\mathrm{M}^{\mathrm{X}} / \mathrm{M} / 1$ queueing process). 
Note that we have defined the $\boldsymbol{Q}$-process as the corresponding transition function $\boldsymbol{P}(t)$ rather than the process itself. In fact, for convenience, we shall freely use this term to denote either of them in this paper. This is, of course, commonly accepted and will not cause any confusion. Since our generator matrix $\boldsymbol{Q}$ is bounded, then, by the general theory of continuous-time Markov chains, we know that there exists only one $\boldsymbol{Q}$-process which is the Feller minimal one. Also note that we have slightly generalized the definition of the generator matrix of a stopped $\mathrm{M}^{\mathrm{X}} / \mathrm{M} / 1$ queue by allowing $\sum_{j \neq 1} b_{j} \leq-b_{1}$. Let

$$
d=-b_{1}-\sum_{j \neq 1} b_{j}
$$

be the deficit. Then $d \geq 0$ and $d=0$ if and only if $Q$ is conservative. The reason in giving this slightly general definition is twofold. Firstly, in our later proof we need to consider the nonconservative case. Secondly, and more importantly, this definition provides us with an opportunity to consider more general models, for example, the so-called $\mathrm{M}^{\mathrm{X}} / \mathrm{M} / 1$ queue with removing working loads. See, for example, [5] and [6].

Since 0 is an absorbing state and $C=\{1,2, \ldots\}$ is an irreducible and transient class for this model, we know that $p_{i j}(t) \rightarrow 0$ as $t \rightarrow \infty$ for all $i, j \in C$. Hence, this process does not possess any limiting distribution in the normal sense. Therefore, we turn our attention to the decay parameter and the related properties, particularly the invariant measures and quasistationary distributions of this stopped $\mathrm{M}^{\mathrm{X}} / \mathrm{M} / 1$ queue.

It should be noted that, for the special case of the stopped $\mathrm{M} / \mathrm{M} / 1$ queue, the decay parameter and the corresponding invariant measures have been obtained; see, for example, [1]. As to the general stopped $M^{X} / M / 1$ queue, Daley [11] discussed the discrete-time version, i.e. the jump chain of the stopped $\mathrm{M}^{\mathrm{X}} / \mathrm{M} / 1$ queue under the further condition that

$$
\sum_{k=1}^{\infty} k b_{k+1}<b_{0} .
$$

In particular, under condition (1.3), Daley [11] obtained the decay parameter of the jump chain, denoted by $R_{C}$, say, and proved that this jump chain is $R_{C}$-transient. The generating function of the quasistationary distribution of this discrete-time jump chain has also been given in [11] under condition (1.3).

Different from Daley [11], in this paper we shall not confine ourselves to condition (1.3). Indeed, all the situations, even including the subtle case in which $\sum_{k=1}^{\infty} k b_{k+1}=+\infty$, shall be discussed in this paper. Many important decay properties which have not been discussed in [11] shall also be revealed. Such properties include, for example, the particularly interesting and important problem of invariant measures and the uniqueness and construction of invariant measures and quasistationary distributions. Also, the main methods used in this paper are substantially different from those used in [11]. Interestingly, our methods are not only applicable to our current more complex and abundant continuous scenario, but are also of methodological significance in their applications to much more general models. For example, our methods are perfectly applicable to the more general $\mathrm{M}^{\mathrm{X}} / \mathrm{M} / 1$ queues with state-dependent control and even to the more general Markovian queueing models. We shall discuss such applications in subsequent papers.

The structure of this paper is as follows. In Section 2 we concentrate on studying the decay parameter for the stopped $\mathrm{M}^{\mathrm{X}} / \mathrm{M} / 1$ queueing process. The exact value of the decay parameter for all cases will be revealed. It shall be shown that this value can be obtained fairly easily 
and, also, has a clear geometric interpretation. In Section 3 we show that the stopped $M^{X} / M / 1$ queueing process is always $\lambda_{C}$-transient, again, for all cases. Some elegant expressions, which in turn reveal some further deep properties of the stopped $\mathrm{M}^{\mathrm{X}} / \mathrm{M} / 1$ queue, are obtained regarding $\lambda_{C}$-transiency. The closed linked $\lambda$-subinvariant vectors and $\lambda$-invariant vectors for $\lambda \in\left[0, \lambda_{C}\right]$ are also presented in this section. The invariant measures and quasistationary distributions are fully discussed in Section 4. We show that there exists a family of $\lambda$-invariant measures and quasistationary distributions, indexed by $\lambda \in\left(0, \lambda_{C}\right]$ except for the (trivial) critical case in which $\lambda_{C}=0$. The explicit expressions for the generating functions of this family of $\lambda$ invariant measures and quasistationary distributions are established. We shall see that these expressions take very simple forms. Finally, in Section 5 several examples are provided to illustrate the results obtained in the previous sections.

\section{Decay parameter}

In order to find the decay parameter $\lambda_{C}$ and to study the invariant measures, invariant vectors, and quasistationary distributions for the stopped $\mathrm{M}^{\mathrm{X}} / \mathrm{M} / 1$ queue, we define $B(s)$ to be the generating function of the given sequence $\left\{b_{k} ; k \geq 0\right\}$, i.e.

$$
B(s)=\sum_{k=0}^{\infty} b_{k} s^{k} .
$$

Since $B(s)$ is a power series, we know that it possesses a convergence radius of

$$
\rho=\frac{1}{\limsup _{n \rightarrow \infty} \sqrt[n]{b_{n}}} .
$$

Clearly, $\rho \geq 1$. By looking back at (1.2) we see that if $\rho$ is finite then, although $B(\rho)=+\infty$ is likely, it is impossible that $B(\rho)=-\infty$. A similar property holds for $B^{\prime}(\rho)$. Now, let $\rho_{0}=\sup \{s>0: B(s) \leq 0\}$.

The following simple lemma summarizes some useful properties of the generating function $B(s)$.

Lemma 2.1. The generating function $B(s)$ is convex in $[0, \rho)$ and has either one or two positive roots. More specifically, the following cases hold.

(i) If $\rho=+\infty$ then $B(s)=0$ has exactly two positive zeros, $q_{S}$ and $q_{L}$, say, satisfying $0<q_{S} \leq q_{L}<\rho$ such that $B(s)>0$ for $s \in\left[0, q_{S}\right) \cup\left(q_{L}, \rho\right)$ and $B(s)<0$ for $s \in\left(q_{S}, q_{L}\right)$. For this case, we have $\rho_{0}=q_{L}, B\left(\rho_{0}\right)=0$, and $0<B^{\prime}\left(\rho_{0}\right) \leq+\infty$, and there exists a point $s_{0}$ with $q_{S}<s_{0}<q_{L}$ such that $B^{\prime}\left(s_{0}\right)=0$. Moreover, $q_{S}=q_{L}=1$ if and only if $d=0$ and $b_{0}=\sum_{j=2}^{\infty}(j-1) b_{j}$, and $q_{S}<1=q_{L}$ if and only if $d=0$ and $b_{0}<\sum_{j=2}^{\infty}(j-1) b_{j} \leq+\infty$.

(ii) If $\rho<+\infty$ and $0 \leq B(\rho) \leq+\infty$ then all the same conclusions hold as in (i).

(iii) If $\rho<+\infty, B(\rho)<0$, and $0 \leq B^{\prime}(\rho) \leq+\infty$, then $B(s)=0$ has exactly one positive root $q_{S} \leq 1$ such that $B(s)>0$ for $s \in\left[0, q_{S}\right)$ and $B(s)<0$ for $s \in\left(q_{S}, \rho\right]$. For this case, we have $\rho_{0}=\rho$ and there exists a point $s_{0}$ satisfying $q_{S}<s_{0} \leq \rho_{0}=\rho$ such that $B^{\prime}\left(s_{0}\right)=0$ and $B^{\prime}(s)<0$ for $s \in\left[0, s_{0}\right)$ and $B^{\prime}(s)>0$ for $s \in\left(s_{0}, \rho_{0}\right]$. Moreover, $s_{0}=\rho_{0}$ if and only if $B^{\prime}(\rho)=0$, while $q_{S}=1$ if and only if $d=0$ and $b_{0} \geq \sum_{j=2}^{\infty}(j-1) b_{j}$. 
(iv) Finally, if $\rho<+\infty, B(\rho)<0$, and $B^{\prime}(\rho)<0$, then $B(s)=0$ has exactly one positive root $q_{S} \leq 1$ such that $B(s)>0$ for $s \in\left(0, q_{S}\right)$ and $B(s)<0$ for $s \in\left(q_{S}, \rho\right]$. For this case, we have $\rho_{0}=\rho$ and $B^{\prime}(s)$ attains its maximum value at $\rho_{0}$ in $[0, \rho]$.

Furthermore, for cases (i)-(iii), we have $B\left(\rho_{0}\right)-\rho_{0} B^{\prime}\left(\rho_{0}\right)<0$, while, for case (iv), either $B\left(\rho_{0}\right)-\rho_{0} B^{\prime}\left(\rho_{0}\right)<0$ or $B\left(\rho_{0}\right)-\rho_{0} B^{\prime}\left(\rho_{0}\right) \geq 0$ may occur.

Proof. Note that we have $B^{\prime \prime}(s)>0$ for all $s \in(0, \rho)$, which is due to (1.2). Thus, $B^{\prime}(s)$ and $B(s)$ are strictly increasing and convex function on $s \in(0, \rho)$, respectively. All the conclusions then easily follow.

Remark 2.1. By Lemma 2.1 we see that both $\rho_{0}$ and $B\left(\rho_{0}\right)$ are finite. In fact, we have $\rho_{0} \in[1,+\infty)$ and $-\infty<B\left(\rho_{0}\right) \leq 0$. Also, there are only two possibilities for $\rho_{0}$ : either $\rho_{0}=q_{L}$ or $\rho_{0}=\rho<+\infty$.

Note that, for cases (i)-(iii) of Lemma 2.1, there exists a tangent line of $B(s)$ which passes through the origin. Even for case (iv) of Lemma 2.1, this may still be true (though not always). We shall prove that, for nearly all the cases (the exact meaning of this will be clear later), the decay parameter is simply the absolute value of the gradient of this tangent line.

Now define

$$
\lambda^{*}=\sup \left\{\lambda \in \mathbb{R}: B(s)+\lambda s=0 \text { has a root in }\left[0, \rho_{0}\right]\right\},
$$

where $\mathbb{R}$ denotes the set of real numbers. Since $B(0)=b_{0}>0 \geq B(1)$, it is easily seen that $\lambda^{*}$ can also be expressed as

$$
\begin{aligned}
\lambda^{*} & =\sup \left\{\lambda \geq 0: B(s)+\lambda s=0 \text { has a root in }\left[0, \rho_{0}\right]\right\} \\
& =\sup \left\{\lambda \geq 0: B(s)+\lambda s=0 \text { has a root in }\left[q_{S}, \rho_{0}\right]\right\} .
\end{aligned}
$$

Indeed, for all $s \in\left[0, q_{S}\right)$ and $\lambda \geq 0$, by Lemma 2.1 we have $B(s)+\lambda s \geq B(s)>0$ and, thus, $B(s)+\lambda s=0$ has no root in $\left[0, q_{S}\right)$.

Later we shall prove that the supreme in (2.1) (or (2.2)) is attainable and, more importantly, that it is just the decay parameter for our model. Hence, it is very informative and useful to give further characteristics of this important quantity, including its geometric meaning. For this purpose, we define

$$
\bar{\lambda}=\max \left\{-\frac{B(s)}{s} ; s \in\left[q_{s}, \rho_{0}\right]\right\} .
$$

Since $-B(s) / s$ is a continuous function on the closed interval $\left[q_{S}, \rho_{0}\right]$ (except the trivial case in which $q_{S}=\rho_{0}$ ), we know that $\bar{\lambda}$ is finite.

In determining the decay parameter the test function

$$
g(s)=B(s)-s B^{\prime}(s)
$$

plays an extremely important role. For this reason, we summarize its simple yet important properties as follows. First note that, as a power series, $g(s)$ has the same convergence radius $\rho$ as $B(s)$.

Lemma 2.2. The test function $g(s)$ is a strictly decreasing function on $[0, \rho)$ and, thus, has either no positive zero or exactly one positive zero, denoted by $s_{*}$, say. Moreover, the former happens if and only if $\rho<+\infty$ and $b_{0}>\sum_{j=2}^{\infty}(j-1) b_{j} \rho^{j}$ (i.e. $\rho<+\infty$ and $g(\rho)>0$ ) and, for this former case, we have $g(s)>0$ for all $s \in[0, \rho]$, while, for the latter case, we have $g(s)>0$ for all $s \in\left[0, s_{*}\right)$ and $g(s)<0$ for all $s \in\left(s_{*}, \rho\right)$. 
Proof. The strictly decreasing property follows directly from the fact that, for all $s>0$, we have $g^{\prime}(s)=-s B^{\prime \prime}(s)<0$. Now all the conclusions easily follow from this strictly decreasing property together with the simple facts that

$$
g(s)=b_{0}-\sum_{j=2}^{\infty}(j-1) b_{j} s^{j} \quad \text { and } \quad g(0)=b_{0}>0 .
$$

Note that $g(s)$ is also actually strictly concave on $[0, \rho)$, but this property will not be used in this paper. The above positive zero, $s_{*}$ of $g(s)$ (if any), plays a key role in considering the decay properties of the stopped $\mathrm{M}^{\mathrm{X}} / \mathrm{M} / 1$ queue as the following lemma and some other later conclusions show.

Lemma 2.3. We have $\lambda^{*}=\bar{\lambda}$ and thus $\lambda^{*}<\infty$, and the supreme in (2.1)( $\left.\operatorname{or}(2.2)\right)$ is attainable. In particular, the equation $B(s)+\lambda^{*} s=0$ has a unique root $s_{*} \in\left[q_{S}, \rho_{0}\right]$, where $q_{S}$ is given in Lemma 2.1. Moreover, the following cases hold.

(i) If $B^{\prime}\left(\rho_{0}\right)>B\left(\rho_{0}\right) / \rho_{0}$ then $\lambda^{*}=-B\left(s_{*}\right) / s_{*}=-B^{\prime}\left(s_{*}\right)$, where $q_{S} \leq s_{*}<\rho_{0}$. For this case, we have $\lambda^{*}>-B^{\prime}\left(\rho_{0}\right)$.

(ii) If $B^{\prime}\left(\rho_{0}\right)=B\left(\rho_{0}\right) / \rho_{0}$ then $\lambda^{*}=-B\left(s_{*}\right) / s_{*}=-B^{\prime}\left(s_{*}\right)$, where $s_{*}=\rho_{0}$; thus, $\lambda^{*}=$ $-B^{\prime}\left(\rho_{0}\right)$.

(iii) If $B^{\prime}\left(\rho_{0}\right)<B\left(\rho_{0}\right) / \rho_{0}$ then $\lambda^{*}=-B\left(s_{*}\right) / s_{*}<-B^{\prime}\left(s_{*}\right)$, where $s_{*}=\rho_{0}=\rho$; thus, $\lambda^{*}<-B^{\prime}\left(\rho_{0}\right)$.

Proof. By Lemma 2.1 we see that $\lambda^{*}$ in (2.1) or (2.2) is well defined and that $\lambda^{*} \geq 0$ since $B(s)=0$ has a root $q_{s} \in\left[0, \rho_{0}\right]$. Now, our aim is to prove that $\lambda^{*}$ is attainable. Firstly, if $B(1)=B^{\prime}(1)=0$ then $q_{s}=\rho_{0}=1$ and, thus, it is trivial to see that all the conclusions hold since $\lambda^{*}=\bar{\lambda}=0$ by definition. For all other cases, $\left[q_{s}, \rho_{0}\right]$ is a closed interval and, thus, by noting that $B(s) / s$ is a nonpositive, continuous function of $s \in\left[q_{s}, \rho_{0}\right]$, we see that there exists an $s_{0} \in\left[q_{s}, \rho_{0}\right]$ such that $\bar{\lambda}=-B\left(s_{0}\right) / s_{0}<+\infty$ and $\bar{\lambda}>0$.

We now claim that $\bar{\lambda}=\lambda^{*}$. Firstly, it is clear that $\bar{\lambda} \leq \lambda^{*}$. Indeed, by the definition of $\bar{\lambda}$ we know that the equation $B(s)+\bar{\lambda} s=0$ has a root $s_{0} \in\left[q_{s}, \rho_{0}\right]$ and, thus, the inequality follows by noting (2.1). In order to prove the converse, we assume that $\bar{\lambda}<\lambda^{*}$. It follows that there exists a $\mu \in\left(\bar{\lambda}, \lambda^{*}\right)$. Since $\mu<\lambda^{*}$, we know that the equation $B(s)+\mu s=0$ has a root $s_{\mu} \in\left[q_{s}, \rho_{0}\right]$, i.e. $\mu=-B\left(s_{\mu}\right) / s_{\mu}$. Hence, $\mu \leq \bar{\lambda}$, since $\bar{\lambda}$ is the maximum of $-B(s) / s$ on $\left[q_{s}, \rho_{0}\right]$, which contradicts $\bar{\lambda}<\mu$. Therefore, for all cases, we have $\lambda^{*}=\bar{\lambda}$. Thus, the first part is proved.

To prove the latter part, let $f(s)=-B(s) / s$ for $s \in\left(0, \rho_{0}\right]$. Considering $f(s)<0$ for $s \in\left[0, q_{S}\right]$, we know that $\lambda^{*}=\max \left\{f(s) ; s \in\left[0, \rho_{0}\right]\right\}$. Now note that $f^{\prime}(s)=g(s) / s^{2}$, where $g(s)$ is defined in (2.3); thus, $f^{\prime}(s)$ shares the same zero and signs as $g(s)$. In particular, $f^{\prime}(s)$ has at most one positive zero. Moreover, if $f^{\prime}(s)$ does have a positive zero, which then must be the same $s_{*}$ as in Lemma 2.2 , then $f(s)$ must attain its maximum on $[0, \rho)$ at $s_{*}$. Indeed, since $f^{\prime}(s)$ and $g(s)$ have the same sign, then, by Lemma $2.2, f(s)$ is strictly increasing on $\left[0, s_{*}\right)$ and strictly decreasing on $\left(s_{*}, \rho\right)$. Therefore, if $B^{\prime}\left(\rho_{0}\right)>B\left(\rho_{0}\right) / \rho_{0}$, i.e. $g\left(\rho_{0}\right)<0$, then there exists an $s_{*}$ satisfying $q_{S}<s_{*}<\rho_{0}$ such that $g\left(s_{*}\right)=f^{\prime}\left(s_{*}\right)=0$. That is, the function $f(s)$ attains its maximum at $s_{*}$ and, hence, $\lambda^{*}=-B\left(s_{*}\right) / s_{*}=-B^{\prime}\left(s_{*}\right)$, where the latter equality holds because of $g\left(s_{*}\right)=0$. It then follows from $g\left(\rho_{0}\right)<0$ that $\lambda^{*}>-B\left(\rho_{0}\right) / \rho_{0}>-B^{\prime}\left(\rho_{0}\right)$ and, thus, (i) is proved. 
Similarly, we may prove (ii) and (iii). The difference is that in both (ii) and (iii) the function $f(s)$ attains its maximum $\lambda^{*}$ at $\rho_{0}$ rather than in $\left(0, \rho_{0}\right)$. Moreover, it is easily seen that in case (iii) we must have $\rho_{0}=\rho$ since, for this case, $\rho_{0}=q_{L}$ is impossible; see Lemma 2.1.

Remark 2.2. (i) Lemma 2.3 shows that the quantity $\lambda^{*}$ has clear geometric interpretation. Indeed, considering that the slope of the tangent line, which passes through the origin, of the curve $y=B(x)$ is just $B(x) / x$, we see that, for both cases (i) and (ii) of Lemma 2.3, $y=-\lambda^{*} x$ is just the tangent line of the curve $y=B(x)$. More intuitively, consider that a family of lines $y=-\lambda x$, indexed by the parameter $\lambda \geq 0$, rotates anticlockwise around the origin. Then $\lambda^{*}$ is just the value of $\lambda$ when the rotating lines $y=-\lambda x(\lambda>0)$ first hit the curve $y=B(x)(x>0)$. Hence, for cases (i) and (ii) of Lemma 2.3, the line $y=-\lambda^{*} x$ is just the tangent line of $y=B(x)$, while, for case (iii) of Lemma 2.3, though not the tangent line, the line $y=-\lambda^{*} x$ and the curve $y=B(x)$ intersect at the point $\left(\rho_{0}, B\left(\rho_{0}\right)\right)$.

(ii) Although in both cases (ii) and (iii) of Lemma 2.3 the function $f(s)=-B(s) / s$ attains its maximum value $\lambda^{*}$ at $\rho_{0}$, they are actually quite different in geometric interpretation. Indeed, for Lemma 2.3(ii), we have $f^{\prime}\left(\rho_{0}\right)=0$ and, thus, $y=-\lambda^{*} x$ is the tangent line of the curve $y=B(x)$, while, for Lemma 2.3(iii), we have $f^{\prime}(s)>0$ for all $s \in\left[0, \rho_{0}\right]$ and, thus, $y=-\lambda^{*} x$ is not the tangent line of the curve $y=B(x)$.

(iii) Note that the same notation $s_{*}$ is used in both Lemmas 2.2 and 2.3. This is reasonable. Indeed, as can be seen from the proof of Lemma 2.3, the root $s_{*}$ given in Lemma 2.3 is the same $s_{*}$ as the positive 0 of $g(s)$ given in Lemma 2.2 when $g(s)$ does have a positive zero. For the case in which $g(s)$ does not possess a positive zero, the $s_{*}$ defined in Lemma 2.3 is just $\rho_{0}=\rho$. It is also clear that case (iii) of Lemma 2.3 can only happen when both $\rho<\infty$ and $b_{0}>\sum_{j=2}^{\infty}(j-1) b_{j} \rho^{j}$ hold. In particular, if $\boldsymbol{Q}$ is conservative then this can only happen when both $\rho<\infty$ and $B^{\prime}(1)<0$ hold.

Lemma 2.4. Let $\left(p_{i j}(t) ; i, j \in \mathbb{Z}_{+}\right)$be a stopped $M^{X} / M / 1$ queueing process with generator matrix $Q$ as defined in (1.1)-(1.2). Then $\lambda_{C} \geq \lambda^{*}$.

Proof. In order to prove that $\lambda_{C} \geq \lambda^{*}$, by Remark (3) of [1, p. 175], we only need to show that there exists a $\lambda^{*}$-subinvariant vector for the minimal transition function $\left(p_{i j}(t) ; i, j \in \mathbb{Z}_{+}\right)$ on $C$, or, equivalently, that there exists a $\lambda^{*}$-subinvariant vector for $\boldsymbol{Q}$ on $C$ (see [1, Proposition 5.4.1]). In other words, we only need to show that there exist $\left(x_{j} ; j \geq 1\right)$ such that $0<x_{j}<$ $+\infty$ for all $j \geq 1$ and

$$
\sum_{j=1}^{\infty} q_{i j} x_{j} \leq-\lambda^{*} x_{i}, \quad i \geq 1
$$

However, in Lemma 2.3 we have proved that the equation $B(s)+\lambda^{*} s=0$ has a unique root $s_{*} \in\left[q_{S}, \rho_{0}\right]$, i.e. $B\left(s_{*}\right)+\lambda^{*} s_{*}=0$. That is,

$$
\sum_{j=0}^{\infty} b_{j} s_{*}^{j}=-\lambda^{*} s_{*}
$$

Now let $x_{j}=s_{*}^{j}(j \geq 1)$. Then $0<x_{j}<+\infty(j \geq 1)$ and it is easily seen that $\left(x_{j} ; j \geq 1\right)$ satisfies (2.4). Indeed, for $i=1,(2.4)$ is just

$$
\sum_{j=1}^{\infty} q_{1 j} x_{j}=\sum_{j=1}^{\infty} b_{j} s_{*}^{j}=B\left(s_{*}\right)-b_{0}=-\lambda^{*} s_{*}-b_{0}<-\lambda^{*} s_{*}=-\lambda^{*} x_{1},
$$


while, for $i \geq 2,(2.4)$ is just

$$
\sum_{j=1}^{\infty} q_{i j} x_{j}=\sum_{j=i-1}^{\infty} b_{j-i+1} s_{*}^{j}=s_{*}^{i-1} B\left(s_{*}\right)=-\lambda^{*} s_{*}^{i}=-\lambda^{*} x_{i} .
$$

Thus, (2.4) holds, which completes the proof.

We are now ready to prove the main result of this section. That is, we shall prove that $\lambda^{*}$ is exactly the decay parameter $\lambda_{C}$, where, again, $C=\{1,2, \ldots\}$ is an irreducible class. We shall prove this basic conclusion in two theorems, dealing with two different cases. We first consider the case in which $\lambda^{*} \geq-B^{\prime}\left(\rho_{0}\right)$. Note that, by Lemma 2.3, this covers cases (i) and (ii) of Lemma 2.3.

Theorem 2.1. For the stopped $M^{X} / M / 1$ queueing process with generator matrix $Q$ as given in (1.1)-(1.2), if $\lambda^{*} \geq-B^{\prime}\left(\rho_{0}\right)$ then $\lambda_{C}=\lambda^{*}$, where $C=\{1,2, \ldots\}$.

Proof. By Lemma 2.4 we only need to show that $\lambda_{C} \leq \lambda^{*}$. We first consider a very special case in which $d=0$ and $b_{0}=\sum_{j=2}^{\infty}(j-1) b_{j}$, and, thus, $B(1)=B^{\prime}(1)=0$. For this case, the corresponding transition function $\left(p_{i j}(t) ; i, j \in \mathbb{Z}_{+}\right)$is honest and $q_{S}=1$ and $\lambda^{*}=0$. Suppose that $\lambda_{C}>\lambda^{*}=0$. Then, for any $\lambda \in\left(0, \lambda_{C}\right)$,

$$
\int_{0}^{\infty} \mathrm{e}^{\lambda t} p_{i 1}(t) \mathrm{d} t<\infty \quad \text { for all } i \geq 1 .
$$

However, by the forward equation we know that $p_{i 0}^{\prime}(t)=b_{0} p_{i 1}(t)$ and, thus,

$$
\int_{0}^{\infty} \mathrm{e}^{\lambda t} p_{i 0}^{\prime}(t) \mathrm{d} t<\infty
$$

Since $\lambda>0$, by using the inequality $\mathrm{e}^{\lambda t} \geq 1+\lambda t$ we obtain

$$
\int_{0}^{\infty} t p_{i 0}^{\prime}(t) \mathrm{d} t<\infty
$$

i.e. $\mathrm{E}_{i}\left[\tau_{0}\right]<+\infty$, where $\tau_{0}$ is the absorbing time to 0 and $\mathrm{E}_{i}$ is the mathematical expectation under the condition that the process starts at state $i$. This contradicts, say, Theorem 2.2 of [6]. Therefore, for this special case, we have $\lambda_{C}=\lambda^{*}=0$.

We now remove the condition that $B(1)=B^{\prime}(1)=0$. Since $\lambda^{*} \geq-B^{\prime}\left(\rho_{0}\right)$, by Lemma 2.3 and Remark 2.2(i), we know that the equation $B(s)+\lambda^{*} s=0$ has a root $s_{*} \in\left[q_{s}, \rho_{0}\right]$ and that $y=-\lambda^{*} s$ is the tangent line of the curve $y=B(s)$. Now, by Lemma 2.3 we also know that the tangency point is just $\left(s_{*}, B\left(s_{*}\right)\right)$ and, hence, $\lambda^{*}=-B^{\prime}\left(s_{*}\right)$. Define $\tilde{\boldsymbol{Q}}=\left(\tilde{q}_{i j} ; i, j \in \mathbb{Z}_{+}\right)$ as

$$
\tilde{q}_{i j}= \begin{cases}\tilde{b}_{j-i+1} & \text { if } i \geq 1, j \geq i-1, \\ 0 & \text { otherwise }\end{cases}
$$

where $\tilde{b}_{k}=b_{k} s_{*}{ }^{k}(k \neq 1)$ and $\tilde{b}_{1}=b_{1} s_{*}+\lambda^{*} s_{*}$. It is obvious that $\tilde{\boldsymbol{Q}}$ is a conservative generator matrix of some stopped $\mathrm{M}^{\mathrm{X}} / \mathrm{M} / 1$ queue. Let $\left(\tilde{p}_{i j}(t) ; i, j \in \mathbb{Z}_{+}\right)$be the unique $\tilde{\boldsymbol{Q}}$-function, and let $\tilde{B}(s)$ denote the generating function of $\left\{\tilde{b}_{k} ; k \geq 0\right\}$, i.e.

$$
\tilde{B}(s)=\sum_{k=0}^{\infty} \tilde{b}_{k} s^{k}, \quad|s| \leq 1 .
$$


It is easy to see that $\tilde{B}(s)=B\left(s_{*} s\right)+\lambda^{*} s_{*} s$ and $\tilde{B}(1)=\tilde{B}^{\prime}(1)=0$. Hence, by the just proven result we know that the decay parameter of $\left(\tilde{p}_{i j}(t) ; i, j \in \mathbb{Z}_{+}\right)$for $C$ is $\tilde{\lambda}_{C}=0$. On the other hand, by Kingman's lemma (see, for example, [1, Theorem 5.2.7 and Proposition 5.4.1]), we know that there exists a $\lambda_{C}$-subinvariant measure for $Q$ on $C$, i.e. there exists a measure $\left(m_{i} ; i \geq 1\right)$ such that $0<m_{i}<\infty(i \geq 1)$ and

$$
\sum_{i=1}^{j+1} m_{i} b_{j-i+1} \leq-\lambda_{C} m_{j}, \quad j \geq 1 .
$$

Multiplying both sides of (2.5) by $s_{*}^{j}$ yields

$$
\sum_{i=1}^{j+1}\left(m_{i} s_{*}{ }^{i-1}\right)\left(b_{j-i+1} s_{*}{ }^{j-i+1}\right) \leq-\left(\lambda_{C} s_{*}\right)\left(m_{j} s_{*}{ }^{j-1}\right), \quad j \geq 1 .
$$

Adding $\lambda^{*} m_{j} s_{*}^{j}$ to both sides of the above inequality and using the definition of $\left\{\tilde{b}_{k} ; k \geq 0\right\}$, immediately yields

$$
\sum_{i=1}^{j+1} \tilde{m}_{i} \tilde{b}_{j-i+1} \leq-\left(\lambda_{C}-\lambda^{*}\right) s_{*} \tilde{m}_{j}, \quad j \geq 1,
$$

where $\tilde{m}_{i}=m_{i} s_{*}{ }^{i-1}(i \geq 1)$. Therefore, $\left(\tilde{m}_{i} ; i \geq 1\right)$ is a $\left(\lambda_{C}-\lambda^{*}\right) s_{*}$-subinvariant measure for $\tilde{\boldsymbol{Q}}$ (or, equivalently, for $\left.\left(\tilde{p}_{i j}(t) ; i, j \in \mathbb{Z}_{+}\right)\right)$on $C$. Now, by a well-known result (see [1, Remarks, p. 175]), we must have $\left(\lambda_{C}-\lambda^{*}\right) s_{*} \leq \tilde{\lambda}_{C}=0$. Hence, $\lambda_{C} \leq \lambda^{*}$ and, therefore, $\lambda_{C}=\lambda^{*}$.

Theorem 2.2, below, shows that even if $\lambda^{*}<-B^{\prime}\left(\rho_{0}\right)$, the same conclusion still holds.

Theorem 2.2. Let $\left(p_{i j}(t) ; i, j \in \mathbb{Z}_{+}\right)$be a stopped $M^{X} / M / 1$ queueing process with generator matrix $Q$ as defined in (1.1)-(1.2). Then $\lambda_{C}=\lambda^{*}$.

Proof. By Theorem 2.1 we only need to consider the case in which $\beta=: B^{\prime}\left(\rho_{0}\right)+\lambda^{*}<$ 0 . Let $\left(\phi_{i j}(\lambda) ; i, j \in \mathbb{Z}_{+}\right)$be the corresponding $Q$-resolvent, i.e. the Laplace transform of $\left(p_{i j}(t) ; i, j \in \mathbb{Z}_{+}\right)$. Now, let $N_{0}=\inf \left\{k \geq 2 ; b_{k}>0\right\}$ and, for any $n \geq N_{0}$, let

$$
q_{i j}^{(n)}= \begin{cases}b_{j-i+1}^{(n)} & \text { if } i \geq 1, j \geq i \\ 0 & \text { otherwise }\end{cases}
$$

where

$$
b_{k}^{(n)}= \begin{cases}b_{k} & \text { if } k \leq n \\ 0 & \text { if } k>n\end{cases}
$$

It is obvious that $\boldsymbol{Q}^{(n)}=\left(q_{i j}^{(n)} ; i, j \in \mathbb{Z}_{+}\right)$is a nonconservative generator matrix as in (1.1)-(1.2) and that $C$ is still an irreducible class for each $\boldsymbol{Q}^{(n)}, n \geq N_{0}$. Let ${ }_{n} p_{i j}(t) ; i, j \in$ $\left.\mathbb{Z}_{+}\right)$and $\left({ }_{n} \phi_{i j}(\lambda) ; i, j \in \mathbb{Z}_{+}\right)$be the Feller minimal $\boldsymbol{Q}^{(n)}$-function and the Feller minimal $\boldsymbol{Q}^{(n)}$-resolvent, respectively. Let

$$
\begin{gathered}
B_{n}(s)=\sum_{k=0}^{\infty} b_{k}^{(n)} s^{k}, \\
\lambda_{n}^{*}=\sup \left\{\lambda \geq 0: B_{n}(s)+\lambda s=0 \text { has a root in }\left[0, \rho_{0}^{(n)}\right]\right\},
\end{gathered}
$$


where $\rho_{0}^{(n)}=\sup \left\{s>0: B_{n}(s) \leq 0\right\}$. It is clear that the generating function $B_{n}(s)$ is well defined on $[0, \infty)$, i.e. the convergence radius $\rho^{(n)}$ for $B_{n}(s)$ is infinite. It follows that $\lambda_{n}^{*} \geq-B_{n}^{\prime}\left(\rho_{0}^{(n)}\right)$. Therefore, we may apply Theorem 2.1 to obtain the conclusion that the decay parameter of $\left(n p_{i j}(t) ; i, j \in \mathbb{Z}_{+}\right)$for $C$ is $\lambda_{C}^{(n)}=\lambda_{n}^{*}$. We now prove that $\lambda_{n}^{*} \downarrow \lambda^{*}(n \uparrow \infty)$, where $\lambda^{*}$ is given in (2.2) with respect to the original generator matrix $\boldsymbol{Q}$. Indeed, by the definition of $B_{n}(s), n \geq N_{0}$, it is clear that

$$
B_{n}(s) \leq B_{n+1}(s), \quad B_{n}^{\prime}(s) \leq B_{n+1}^{\prime}(s), \quad \text { for all } s \geq 0, n \geq N_{0},
$$

and that

$$
B_{n}(s) \leq B(s), \quad B_{n}^{\prime}(s) \leq B^{\prime}(s), \quad \text { for all } 0 \leq s<\rho, n \geq N_{0} .
$$

In other words, the curve $y=B_{n+1}(s)$ is above the curve $y=B_{n}(s)$ for $s \geq 0$. Since $\lambda_{n}^{*}$ and $\lambda_{n+1}^{*}$ are the absolute values of the slopes of the tangent lines, passing through the origin, of the curves $y=B_{n}(s)$ and $y=B_{n+1}(s)$, respectively, it follows from (2.6) and (2.7) that we must have $\lambda_{n}^{*} \geq \lambda_{n+1}^{*}\left(n \geq N_{0}\right)$, i.e. $\left\{\lambda_{n}^{*} ; n \geq N_{0}\right\}$ is nonincreasing with respect to $n$, and it is also clear that $\lambda_{n}^{*} \geq \lambda^{*}\left(n \geq N_{0}\right)$. Let $\bar{\lambda}=\lim _{n \rightarrow \infty} \lambda_{n}^{*}$. Then $\bar{\lambda} \geq \lambda^{*}$. We now claim that $\bar{\lambda}=\lambda^{*}$. Suppose that $\bar{\lambda}>\lambda^{*}$, then choose $\tilde{\lambda} \in\left(\lambda^{*}, \bar{\lambda} \wedge\left(\lambda^{*}-\beta\right)\right)$. Since, for each $n \geq N_{0}, B_{n}(s)+\tilde{\lambda} s$ is strictly convex in $[0, \infty)$, there exists a unique $\tilde{s}_{n}$ such that $B_{n}\left(\tilde{s}_{n}\right)+\tilde{\lambda} \tilde{s}_{n}=\inf \left\{B_{n}(s)+\tilde{\lambda} s: s \in[0, \infty)\right\}$. It can be easily seen that $\left\{\tilde{s}_{n} ; n \geq N_{0}\right\}$ is nonincreasing with respect to $n$. Indeed, since the function $B_{n}(s)+\tilde{\lambda} s$ attains its minimum value at $\tilde{s}_{n}$ and $B_{n}^{\prime}(s)+\tilde{\lambda}$ is differentiable at $s=\tilde{s}_{n}$, we must have $B_{n}^{\prime}\left(\tilde{s}_{n}\right)=-\tilde{\lambda}$. However, by (2.6), $B_{n+1}^{\prime}\left(\tilde{s}_{n}\right) \geq B_{n}^{\prime}\left(\tilde{s}_{n}\right)=-\tilde{\lambda}$ and, furthermore, for all $s \geq \tilde{s}_{n}, B_{n+1}^{\prime}(s)+\tilde{\lambda} \geq 0$ (since $B_{n+1}^{\prime}(s)$ is an increasing function of $\left.s \in[0, \infty)\right)$. Therefore, the function $B_{n+1}(s)+\tilde{\lambda} s$ is increasing on $\left[\tilde{s}_{n}, \infty\right)$ and, thus, it can only attain its minimum value before $\tilde{s}_{n}$, i.e. $\tilde{s}_{n+1} \leq \tilde{s}_{n}$ for all $n \geq N_{0}$. For the same reason (see (2.7)), we have

$$
B_{n}^{\prime}(s)+\tilde{\lambda} \leq B^{\prime}(s)+\tilde{\lambda} \quad \text { for all } s \in[0, \rho), n \geq N_{0},
$$

and, in particular,

$$
B_{n}^{\prime}\left(\rho_{0}\right)+\tilde{\lambda} \leq B^{\prime}\left(\rho_{0}\right)+\tilde{\lambda}=\beta+\tilde{\lambda}-\lambda^{*}<0 .
$$

But, $B_{n}^{\prime}\left(\tilde{s}_{n}\right)+\tilde{\lambda}=0$ and $B_{n}^{\prime}(s)+\tilde{\lambda}$ is increasing with respect to $s$ and, therefore, $\tilde{s}_{n}>\rho_{0}(n \geq$ $\left.N_{0}\right)$.

Since $\tilde{\lambda}<\bar{\lambda}$, we may claim that

$$
B_{n}\left(\tilde{s}_{n}\right)+\tilde{\lambda} \tilde{s}_{n}<0<B\left(\rho_{0}\right)+\tilde{\lambda} \rho_{0} .
$$

Indeed, since $\tilde{\lambda}>\lambda^{*}$, the equation $B(s)+\tilde{\lambda} s=0$ would have no root in $\left[0, \rho_{0}\right]$ and, thus, would keep positive on $\left[0, \rho_{0}\right]$, which yields the right-hand side inequality of (2.8). As to the left-hand side inequality of (2.8), just note that $\lambda_{n}^{*}$ is the largest value of $\lambda$ such that $B_{n}(s)+\lambda s=0$ has a root in $\left[0, \rho_{0}^{(n)}\right]$. On the other hand, note that $\tilde{\lambda}<\bar{\lambda} \leq \lambda_{n}^{*}$, we know that $B_{n}(s)+\tilde{\lambda} s=0$ has a root in $\left[0, \rho_{0}^{(n)}\right]$ and, thus, the minimum value of $B_{n}(s)+\tilde{\lambda} s$, which is $B_{n}\left(\tilde{s}_{n}\right)+\tilde{\lambda} \tilde{s}_{n}$, must be negative. This yields the inequality on the left-hand side of (2.8).

By the definition of $B_{n}(s)$ we see that the left-hand side inequality of (2.8) can be written as

$$
\sum_{k \neq 1} b_{k}^{(n)} \tilde{s}_{n}^{k}+\tilde{\lambda} \tilde{s}_{n}<-b_{1} \tilde{s}_{n} .
$$


Note that $\tilde{s}_{n} \downarrow \tilde{s}_{\infty}$, say, as $n$ tends to $\infty$, and, thus, by (2.9) we have

$$
\limsup _{n \rightarrow \infty} \sum_{k \neq 1} b_{k}^{(n)} \tilde{s}_{n}^{k}+\tilde{\lambda} \tilde{s}_{\infty} \leq-b_{1} \tilde{s}_{\infty} .
$$

Now considering that the left-hand side of (2.10) is a nonnegative series and that, for each fixed $k \neq 1$, we have

$$
\lim _{n \rightarrow \infty} b_{k}^{(n)} \tilde{s}_{n}^{k}=b_{k} \tilde{s}_{\infty}^{k}
$$

then using Fatou's lemma, (2.10) becomes

$$
\sum_{k \neq 1} b_{k} \tilde{s}_{\infty}^{k}+\tilde{\lambda} \tilde{s}_{\infty} \leq-b_{1} \tilde{s}_{\infty}
$$

which leads to

$$
B\left(\tilde{s}_{\infty}\right) \leq-\tilde{\lambda} \tilde{s}_{\infty}<0 .
$$

However, $\rho_{0}=\sup \{s>0: B(s) \leq 0\}$ and, thus, (2.11) implies that $\tilde{s}_{\infty} \leq \rho_{0}$. But, for all $n \geq N_{0}$, we have $\tilde{s}_{n}>\rho_{0}$ and, thus, $\tilde{s}_{\infty} \geq \rho_{0}$. Therefore, $\tilde{s}_{\infty}=\rho_{0}$. Now, by (2.8) and (2.11),

$$
B\left(\rho_{0}\right)+\tilde{\lambda} \rho_{0} \leq 0<B\left(\rho_{0}\right)+\tilde{\lambda} \rho_{0},
$$

which is a contradiction. This proves that $\bar{\lambda}=\lambda^{*}$.

On the other hand, it is well known that the $Q$-resolvent $\left(\phi_{i j}(\lambda) ; i, j \in \mathbb{Z}_{+}\right)$is the minimal nonnegative solution of the Kolmogorov backward equation,

$$
\phi_{i j}(\lambda)=\frac{\delta_{i j}}{\lambda+q_{i}}+\sum_{k \neq i} \frac{q_{i k}}{\lambda+q_{i}} \phi_{k j}(\lambda), \quad i \geq 0,
$$

and that the $\boldsymbol{Q}^{(n)}$-resolvent $\left({ }_{n} \phi_{i j}(\lambda) ; i, j \in \mathbb{Z}_{+}\right.$) is the minimal nonnegative solution of the Kolmogorov backward equation,

$$
{ }_{n} \phi_{i j}(\lambda)=\frac{\delta_{i j}}{\lambda+q_{i}^{(n)}}+\sum_{k \neq i} \frac{q_{i k}^{(n)}}{\lambda+q_{i}^{(n)}}{ }^{n} \phi_{k j}(\lambda), \quad i \geq 0
$$

and that all of them can be obtained by using the well-known iteration scheme. Now note that $q_{i}^{(n)}=q_{n}\left(n \geq N_{0}\right)$ and $q_{i k}^{(n)} \uparrow q_{i k}(n \uparrow \infty)$ for all $i \neq k$. It is easily seen, by considering their iteration schemes, that ${ }_{n} \phi_{i j}(\lambda) \uparrow \phi_{i j}(\lambda)$ as $n \uparrow \infty$ and, thus, for their corresponding transition functions, we also have ${ }_{n} p_{i j}(t) \uparrow p_{i j}(t)$ as $n \uparrow \infty$.

Now if $\lambda_{C}>\lambda^{*}$ then, for any $\mu \in\left(\lambda^{*}, \lambda_{C}\right)$, we have

$$
\int_{0}^{\infty} \mathrm{e}^{\mu t} p_{11}(t) \mathrm{d} t<+\infty
$$

and, thus,

$$
\int_{0}^{\infty} \mathrm{e}^{\mu t}{ }_{n} p_{11}(t) \mathrm{d} t<+\infty \text { for all } n \geq N_{0} .
$$

It follows from the above proof that $\mu \leq \lambda_{n}^{*}\left(n \geq N_{0}\right)$. Hence, $\mu \leq \lambda^{*}$, which contradicts $\mu \in\left(\lambda^{*}, \lambda_{C}\right)$. Therefore, $\lambda_{C}=\lambda^{*}$. The proof is complete. 
By combining Theorem 2.2 and Lemma 2.3, we obtain the following effective way to obtain the exact value of the decay parameter $\lambda_{C}$.

Corollary 2.1. Let $\rho_{0}$ be determined as in Remark 2.1, and let $g(s)$ be as given in (2.3).

(i) If $g\left(\rho_{0}\right) \geq 0$ then $\rho_{0}=\rho$ and $\lambda_{C}=-B(\rho) / \rho$.

(ii) If $g\left(\rho_{0}\right)<0$ (thus, $g(s)=0$ has a unique root $\left.s_{*} \in\left[0, \rho_{0}\right)\right)$ then $\lambda_{C}=-B^{\prime}\left(s_{*}\right)$ and, hence, $s_{*}$ and $\lambda_{C}$ satisfy the equations

$$
\begin{gathered}
B\left(s_{*}\right)-s_{*} B^{\prime}\left(s_{*}\right)=0, \\
\lambda_{C}=-B^{\prime}\left(s_{*}\right) .
\end{gathered}
$$

In other words, $s_{*}$ and $\lambda_{C}$ are the unique solution of the following equations regarding the unknowns $s$ and $\lambda$ :

$$
\begin{gathered}
B(s)-s B^{\prime}(s)=0, \\
\lambda=-B^{\prime}(s) .
\end{gathered}
$$

Proof. This is a direct consequence of Theorem 2.2 and Lemma 2.3. Also, the uniqueness of the solution of (2.13) can be easily proved.

In applying Corollary 2.1 we need to check the sign of $g\left(\rho_{0}\right)$, which may not always be convenient. This is because $\rho_{0}$ may equal the largest root $q_{L}$ of $B(s)=0$; see Lemma 2.1. But, it may not always be easy to find $q_{L}$. Fortunately, this difficulty can be avoided. In fact, we actually do not need to find $q_{L}$. Indeed, as shown in Lemma 2.1, for cases (i)-(iii) of Lemma 2.1, we automatically have $g\left(\rho_{0}\right)<0$ and, thus, only in case (iv) of Lemma 2.1 do we need to check whether this condition holds, as Corollary 2.2, below, shows. The basic feature of Corollary 2.2 is that all the conditions are imposed to the easily obtained quality $\rho$ rather than $\rho_{0}$. Recall that $\rho$ is the convergence radius of $B(s)$. In Corollary 2.2, we shall only be concerned with the conservative case, since this is the most important case. Also, for the nonconservative case, similar statements can be easily given.

Corollary 2.2. Let $\boldsymbol{Q}$ be a conservative generator matrix as defined in (1.1)-(1.2), and let $B(s)$ be the generating function of the sequence $\left\{b_{k} ; k \geq 0\right\}$, with convergence radius $\rho$.

(i) If $B^{\prime}(1)<0$ and $\rho=1$ or if $B^{\prime}(1)=0$, then $\lambda_{C}=0$ and $s_{*}=1$.

(ii) If $B^{\prime}(1)>0$ (including $\left.B^{\prime}(1)=+\infty\right)$ then $0<s_{*}<1$ and $\lambda_{C}>0$. Moreover, $s_{*}$ and $\lambda_{C}$ can be determined by solving either (2.12) or (2.13) directly.

(iii) If $B^{\prime}(1)<0$ and $\rho>1$ (including $\rho=+\infty$ ) then $s_{*}>1$ and $\lambda_{C}>0$. Moreover, if $\rho<+\infty, B^{\prime}(\rho)<0$, and $B(\rho)>\rho B^{\prime}(\rho)$, then $s_{*}=\rho$ and $\lambda_{C}=-B(\rho) / \rho$, while if any one of the above conditions fails, then $s_{*}$ and $\lambda_{C}$ can be determined by solving either (2.12) or (2.13) directly.

Proof. This is a direct consequence of Lemma 2.1 and Corollary 2.1.

Remark 2.3. Corollary 2.2 tells us that only for the case in which $B^{\prime}(1)<0$ and $\rho>1$ do we need to check whether the condition $B(\rho)>\rho B^{\prime}(\rho)$ is satisfied or not. Furthermore, for Corollary 2.2(iii), even if $\rho=+\infty$ or even if $\rho<+\infty$ with $B(\rho) \geq 0$ or $B^{\prime}(\rho) \geq 0$, then we may immediately claim that $\lambda_{C}$ and $s_{*}$ can be determined by solving (2.12) or (2.13) directly. Furthermore, note that even for the worst case we still may not have to calculate the exact values of $B(\rho)$ and $B^{\prime}(\rho)$, since what we only need to know is whether $B(\rho)>\rho B^{\prime}(\rho)$ or not. In 
Section 5 we shall use several examples to show how easily Corollary 2.2 can be applied to find the decay parameter of the corresponding models.

Corollary 2.3, below, on the other hand, shows that our basic result regarding the decay parameter has a very clear geometric interpretation.

Corollary 2.3. Let $\left(p_{i j}(t) ; i, j \in \mathbb{Z}_{+}\right)$be the stopped $M^{X} / M / 1$ queueing process with generator matrix $\boldsymbol{Q}$ as defined in (1.1)-(1.2).

(i) If $-B\left(\rho_{0}\right) / \rho_{0}<\max \left\{-B(s) / s: s \in\left[q_{S}, \rho_{0}\right]\right\}$ then $y=-\lambda_{C} x$ is the tangent line of the curve $y=B(x)$.

(ii) If $-B\left(\rho_{0}\right) / \rho_{0}=\max \left\{-B(s) / s: s \in\left[q_{S}, \rho_{0}\right]\right\}$ then $\lambda_{C}=-B\left(\rho_{0}\right) / \rho_{0}$.

Finally, we point out that, as a direct corollary, we may also obtain the decay parameter $R_{C}$ of the corresponding jump Markov chain $\left\{X_{n} ; n \geq 0\right\}$. To this end, let $C=\{1,2, \ldots\}$, then it is easy to see that $C$ is also an irreducible class of the jump chain, which possesses the decay parameter $R_{C}$. Also, let $f(s)=s+B(s) / b$ and let $m=f^{\prime}(1)=1+B^{\prime}(1) / b$, where $b=-b_{1}>0$. Obviously, $m<1$ if and only if $B^{\prime}(1)<0$. Furthermore, let $s_{*}$ be the unique positive root (if any) of $s B^{\prime}(s)-B(s)=0$ or, equivalently, let $s_{*}$ be the unique positive root (if any) of $s f^{\prime}(s)-f(s)=0$. Then we have the following conclusion.

Corollary 2.4. Suppose that $\boldsymbol{Q}=\left(q_{i j} ; i, j \in \mathbb{Z}_{+}\right)$is a conservative generator matrix as defined in (1.1)-(1.2) satisfying $b_{0}>0$ and $\sum_{j=2}^{\infty} b_{j}>0$. Then $C=\{1,2, \ldots\}$ is an irreducible class of the jump chain $\left\{X_{n} ; n \geq 0\right\}$.

(i) If $m=1$ or if $m<1$ and $\rho=1$, then $R_{C}=1$ and $s_{*}=1$.

(ii) If $m>1$ (including $m=+\infty$ ) then $0<s_{*}<1$ and $R_{C}=s_{*} / f\left(s_{*}\right)$.

(iii) If $m<1$ and $\rho>1$ (including $\rho=+\infty$ ) then $s_{*}>1$. Moreover, if $\rho<+\infty, f^{\prime}(\rho)<1$, and $f(\rho)>\rho f^{\prime}(\rho)$, then $s_{*}=\rho$ and $R_{C}=\rho / f(\rho)$, while if any one of the above conditions fails then $R_{C}=s_{*} / f\left(s_{*}\right)$.

Proof. By a well-known result of [39] regarding the relationship between the decay parameters of a continuous-time Markov chain and its jump chain, we know that

$$
R_{C}=\left(1-\frac{\lambda_{C}}{b}\right)^{-1}
$$

Now, applying Cororollary 2.2 together with some easy algebra, we immediately yield all the conclusions.

Note that Corollary 2.4(iii) coincides with the basic result obtained in [11]. In fact, Daley [11] only considered the case in which $m<1$.

\section{Invariant vectors and the transiency property}

From now on, we shall assume that the $q$-matrix $\boldsymbol{Q}$ is conservative. After obtaining the exact value of the decay parameter $\lambda_{C}$ in the previous section, we are now interested in realizing whether the stopped $\mathrm{M}^{\mathrm{X}} / \mathrm{M} / 1$ queue process is $\lambda_{C}$-transient or not. We are also interested in some other related properties, particularly the closely linked concept of invariant vectors. However, the answer to the former question is a direct consequence of Lemma 2.4 and Theorem 2.2. Indeed, we have the following conclusion. 
Theorem 3.1. The stopped $M^{X} / M / 1$ queue is always $\lambda_{C}$-transient.

Proof. By Theorem 2.2 we know that $\lambda_{C}=\lambda^{*}$, where $\lambda^{*}$ is given in (2.1), which implies, by Lemma 2.4, that the $\lambda^{*}$-subinvariant vector given there is just a $\lambda_{C}$-subinvariant vector for $\boldsymbol{Q}$ on $C$. However, this $\lambda_{C}$-subinvariant vector is not $\lambda_{C}$-invariant and, thus, by [39] we conclude that the stopped $\mathrm{M}^{\mathrm{X}} / \mathrm{M} / 1$ queue is $\lambda_{C}$-transient.

Remark 3.1. In [39] the conclusion is in terms of invariant measures. However, it is easily seen that the conclusion holds well if the invariant measures are replaced by invariant vectors.

The disadvantage of Theorem 3.1 is that it does not provide sufficient information regarding the transiency property and, thus, is not particularly interesting. Fortunately, we can do much better than Theorem 3.1 by giving further interesting and very useful expressions.

To achieve this aim, we need to carry out some preparatory work. First note that, by the results obtained in the previous section, we know that there exists an $s_{*} \in\left[q_{S}, \rho_{0}\right]$ such that $\lambda_{C}=-B\left(s_{*}\right) / s_{*}$. In particular, for cases (ii) and (iii) of Corollary 2.2, we have $\lambda_{C}>0$ and, for any $\lambda \in\left(0, \lambda_{C}\right]$, the equation $B(s)+\lambda s=0$ has either one root or two roots in $(0, \rho]$ (of course, if $\rho=+\infty$ then this semiclosed interval should be read as $(0, \infty))$, since $B(s)+\lambda s$ is a convex function of $s \in[0, \rho]$. In particular, for any $\lambda \in\left(0, \lambda_{C}\right]$, the equation $B(s)+\lambda s=0$ possesses a smallest positive root. From now on, we shall always use $s_{\lambda}$ to denote this smallest positive root and, hence, if $\lambda=\lambda_{C}$ then $s_{\lambda}=s_{*}$. Moreover, by Lemma 2.3 of [6], for any $\lambda \in(-\infty, 0)$, the equation $B(s)+\lambda s=0$ has exactly one root, denoted also by $s_{\lambda}$, on $[0,1]$. Lemma 3.1, below, reveals further properties of this function which will be useful in our future analysis. Note that we may view $s_{\lambda}$ as a function of $\lambda \in\left(-\infty, \lambda_{C}\right]$. For convenience, we shall freely interchange the notation $s_{\lambda}$ and $S(\lambda)$ in the following. Also, for notational convenience, we shall simply use $\lim _{\lambda \rightarrow \lambda_{C}}$ to denote $\lim _{\lambda \rightarrow \lambda_{C}}{ }^{-}$.

Lemma 3.1. The smallest positive root $S(\lambda)$ of the equation $B(s)+\lambda s=0$, viewed as a function of $\lambda \in\left(-\infty, \lambda_{C}\right]$, possesses the following properties.

(i) $S(\lambda) \in C^{\infty}\left(-\infty, \lambda_{C}\right)$.

(ii) $S(\lambda)$ is a strictly increasing and continuous function of $\lambda \in\left[0, \lambda_{C}\right]$.

(iii) $S(\lambda) \rightarrow 0$ as $\lambda \rightarrow-\infty$ and $S(\lambda) \rightarrow s_{*}$ as $\lambda \rightarrow \lambda_{C}$.

(iv) If $B^{\prime}(1)<0$ and $\rho>1$ then $S(0)=1$ and, for $\lambda \in(-\infty, 0)$, we have $0<S(\lambda)<1$ and, for $\lambda \in(0, \lambda)$, we have $1<S(\lambda)<s_{*}$. Whereas if $B^{\prime}(1)>0$ (including $\left.B^{\prime}(1)=+\infty\right)$ then $S(0)=q<1$ and, for $\lambda \in(-\infty, 0)$, we have $0<S(\lambda)<q$ and, for $\lambda \in\left(0, \lambda_{C}\right)$, we have $q<S(\lambda)<s_{*}<1$.

(v) If $B^{\prime}(1)=0$ or if $B^{\prime}(1)<0$ and $\rho=1$, then $\lambda_{C}=0$ and $s_{*}=1$; thus, $S(0)=S\left(\lambda_{C}\right)$ $=1$ and, for $\lambda \in(-\infty, 0)$, we have $0<S(\lambda)<1$.

(vi) $\lim _{\lambda \rightarrow \lambda_{C}} S^{\prime}(\lambda)=s_{*}^{2} /\left(B\left(s_{*}\right)-s_{*} B^{\prime}\left(s_{*}\right)\right)$.

$$
\lim _{\lambda \rightarrow \lambda_{C}} S^{\prime \prime}(\lambda)=\frac{s_{*}^{3}}{\left(B\left(s_{*}\right)-s_{*} B^{\prime}\left(s_{*}\right)\right)^{2}}\left(2+\frac{s_{*}^{2} B^{\prime \prime}\left(s_{*}\right)}{B\left(s_{*}\right)-s_{*} B^{\prime}\left(s_{*}\right)}\right) .
$$

Proof. Note that $S(\lambda)$ can be viewed as the $x$-coordinate of the intersection point of the curve $y=B(x)$ and the line $y=-\lambda x$; thus, properties (ii) and (iii) immediately follow since the former curve is convex for $x \geq 0$. Properties (iv) and (v) can be easily proven and, in fact, have 
been mentioned before. Now, $S(\lambda)$ is the smallest positive root of the equation $\lambda=-B(s) / s$ and so $\lambda$, as a function of $s \in(0, \rho)$, belongs to $C^{\infty}$, since $B(s)$ is a power series. Hence, the inverse function $S(\cdot)$ also belongs to $C^{\infty}\left(-\infty, \lambda_{C}\right)$, which proves (i).

By considering $S(\lambda)$ to be the root of $B(s)+\lambda s=0$ we have $B(S(\lambda))+\lambda S(\lambda) \equiv 0$. Using (i), we may differentiate the above equation with respect to $\lambda$ to obtain

$$
B^{\prime}(S(\lambda)) S^{\prime}(\lambda)+S(\lambda)+\lambda S^{\prime}(\lambda)=0 .
$$

Letting $\lambda \rightarrow \lambda_{C}{ }^{-}$, using (iii) and the fact that $\lambda_{C}=-B\left(s_{*}\right) / s_{*}$, immediately yields (vi). Similarly, applying (i) and differentiating once again for $\lambda \in\left(-\infty, \lambda_{C}\right)$, we may easily obtain (vii). The proof is complete.

By the proof of Lemma 3.1 we see that the limits of the higher derivatives of $S(\lambda)$ as $\lambda \rightarrow \lambda_{C}$ could be similarly obtained.

We are now in the position to claim our conclusion regarding the subinvariant and invariant vectors of the stopped $\mathrm{M}^{\mathrm{X}} / \mathrm{M} / 1$ queue process.

Theorem 3.2. For any $0 \leq \lambda \leq \lambda_{C}$, let $s_{\lambda}$ be the smallest nonnegative zero of $B(s)+\lambda s$. Then the vector $\boldsymbol{y}=\left(y_{j} ; j \in C\right)$, where $y_{j}=s_{\lambda}^{j}(j \geq 1)$ is a $\lambda$-subinvariant vector for $\boldsymbol{Q}$ as well as for the $\boldsymbol{Q}$-function $\boldsymbol{P}(t)$ on $C$. Similarly, the vector $\overline{\boldsymbol{y}}=\left(\overline{\boldsymbol{y}}_{j} ; j \in C\right)$, where $\bar{y}_{j}=j s_{\lambda}^{j-1}$ $(j \geq 1)$ is a $\lambda$-subinvariant vector for $\boldsymbol{Q}$ as well as for $\boldsymbol{P}(t)$ on $C$ and this $\overline{\boldsymbol{y}}=\left(\bar{y}_{j} ; j \in C\right)$ becomes a $\lambda$-invariant for $\boldsymbol{Q}$ as well as for $\boldsymbol{P}(t)$ on $C$ if and only if $g\left(\rho_{0}\right) \leq 0$ and $\lambda=\lambda_{C}$.

Proof. The proof of the first assertion is very similar to that given in Lemma 2.4. Hence, we only need to prove the second assertion.

Suppose that $0 \leq \lambda \leq \lambda_{C}$. By combining the fact that $\lambda=-B\left(s_{\lambda}\right) / s_{\lambda}$ with the fact that $g\left(s_{\lambda}\right) \geq 0$, where the latter inequality follows from Lemma 2.2 and Lemma 3.1(ii), we obtain $B^{\prime}\left(s_{\lambda}\right) \leq B\left(s_{\lambda}\right) / s_{\lambda}=-\lambda$. It then follows that, for $i=1$,

$$
\sum_{j=1}^{\infty} q_{1 j} \bar{y}_{j}=\sum_{j=1}^{\infty} b_{j} j s_{\lambda}^{j-1}=B^{\prime}\left(s_{\lambda}\right) \leq-\lambda=-\lambda \bar{y}_{1},
$$

and that, for any $i \geq 2$,

$$
\begin{aligned}
\sum_{j=1}^{\infty} q_{i j} \bar{y}_{j} & =\sum_{j=i-1}^{\infty} b_{j-i+1} j s_{\lambda}^{j-1} \\
& =s_{\lambda}^{i-2}\left(\sum_{k=1}^{\infty} k b_{k} s_{\lambda}^{k}+(i-1) \sum_{k=0}^{\infty} b_{k} s_{\lambda}^{k}\right) \\
& =s_{\lambda}^{i-2}\left(B^{\prime}\left(s_{\lambda}\right) s_{\lambda}-B\left(s_{\lambda}\right)\right)+i s_{\lambda}^{i-1} \frac{B\left(s_{\lambda}\right)}{s_{\lambda}} \\
& \leq i s_{\lambda}^{i-1} \frac{B\left(s_{\lambda}\right)}{s_{\lambda}} \\
& =-\lambda \bar{y}_{i}
\end{aligned}
$$

which implies that $\left(j s_{\lambda}^{j-1} ; j \geq 1\right)$ is a $\lambda$-subinvariant vector for $\boldsymbol{Q}$ on $C$. Moreover, it is easily seen that both of the above inequalities become equalities if and only if $B^{\prime}\left(s_{\lambda}\right)=-\lambda$ which, by Lemmas 2.2 and 2.3, is equivalent to $g\left(\rho_{0}\right) \leq 0$ and $\lambda=\lambda_{C}$. 
Finally, noting the facts that $\left(x_{j} ; j \in C\right)$ is a $\mu$-subinvariant vector for $\boldsymbol{Q}$ on $C$ if and only if $\left(x_{j} ; j \in C\right)$ is a $\mu$-subinvariant vector for the minimal $\boldsymbol{Q}$-function $\boldsymbol{P}(t)$ on $C$ and that, for a bounded $q$-matrix $Q,\left(x_{j} ; j \in C\right)$ is a $\mu$-invariant vector for $Q$ on $C$ if and only if $\left(x_{j} ; j \in C\right)$ is a $\mu$-invariant vector for the minimal $\boldsymbol{Q}$-function $\boldsymbol{P}(t)$ on $C$, all of the conclusions follow. The proof is complete.

We point out that if $B^{\prime}(1)<0$ then the discrete-time version of Theorem 3.2 coincides with Theorem 4 of [11].

The following conclusion is our main result in this section.

Theorem 3.3. Let $\left(p_{i j}(t) ; i, j \in \mathbb{Z}_{+}\right)$be the stopped $M^{X} / M / 1$ queueing process with generator matrix $Q$ as defined in (1.1)-(1.2). Then, for any $\lambda \in\left(-\infty, \lambda_{C}\right]$ and $i \geq 1$,

$$
\int_{0}^{\infty} \mathrm{e}^{\lambda t} p_{i 0}^{\prime}(t) \mathrm{d} t=s_{\lambda}^{i}
$$

and

$$
\sum_{j=1}^{\infty}\left(\int_{0}^{\infty} \mathrm{e}^{\lambda t} p_{i j}(t) \mathrm{d} t\right) s^{j-1}=\frac{s_{\lambda}^{i}-s^{i}}{B(s)+\lambda s}, \quad|s|<s_{\lambda},
$$

where $s_{\lambda}$ is the smallest positive root of $B(s)+\lambda s=0$. Moreover,

$$
\int_{0}^{\infty} \mathrm{e}^{\lambda t} p_{i j}(t) \mathrm{d} t=s_{\lambda}^{i+1-j} \sum_{k=0}^{(j-1) \wedge(i-1)} \frac{G_{\lambda}^{(j-k-1)}(0)}{(j-k-1) !}, \quad j \geq 1,
$$

where $G_{\lambda}^{(k)}(0)$ denotes the kth degree derivative of $G_{\lambda}(s)=1-s /\left(B\left(s_{\lambda} s\right)+\lambda s_{\lambda} s\right)$ evaluated at 0 . In particular, the stopped $M^{X} / M / 1$ queue process is always $\lambda_{C}$-transient.

Proof. Firstly, for any $\lambda<0$, (3.1)-(3.3) directly follow from Equations (2.24), (2.27), and (2.29) of [6]. We now prove that (3.1)-(3.3) still hold for $0 \leq \lambda \leq \lambda_{C}$. Indeed, for given $\lambda \in\left[0, \lambda_{C}\right]$, we have shown in Theorem 3.2 that there exists a $\lambda$-subinvariant vector $\left(s_{\lambda}^{j} ; j \geq 1\right)$ for the $Q$-function $\left(p_{i j}(t) ; i, j \geq 0\right)$ on $C$. Now, let

$$
\begin{array}{ll}
\bar{p}_{i j}(t)=\mathrm{e}^{\lambda t} p_{i j}(t) s_{\lambda}^{j-i}, & i, j \geq 1, \\
\bar{p}_{i 0}(t) & =1-\sum_{j=1}^{\infty} \bar{p}_{i j}(t), \quad i \geq 1,
\end{array}
$$

and

$$
\bar{p}_{0 j}(t)=\delta_{0 j}, \quad j \geq 0 .
$$

Then it is easily seen that $\overline{\boldsymbol{P}}(t)=\left(\bar{p}_{i j}(t) ; i, j \geq 0\right)$ is an honest transition function defined on $\mathbb{Z}_{+}$whose $q$-matrix $\overline{\boldsymbol{Q}}=\left(\bar{q}_{i j} ; i, j \geq 0\right)$ is given by

$$
\bar{q}_{i j}= \begin{cases}\bar{b}_{j-i+1} & \text { if } i \geq 1, j \geq i-1 \\ 0 & \text { otherwise }\end{cases}
$$

where $\bar{b}_{j}=b_{j} s_{\lambda}^{j-1}(j \neq 1)$ and $\bar{b}_{1}=\lambda+b_{1}$. Obviously, $\bar{Q}$ is a conservative $q$-matrix of the 
same type as the stopped $\mathrm{M}^{\mathrm{X}} / \mathrm{M} / 1$ queue in (1.1)-(1.2) and, thus, the structure of its resolvent is revealed in [6]. In particular, by Equations (2.24), (2.27), and (2.29) of [6], we have, for any $|s| \leq 1$ and $\mu>0$,

$$
\begin{aligned}
\sum_{j=0}^{\infty}\left(\int_{0}^{\infty} \mathrm{e}^{-\mu t} \bar{p}_{i j}(t) \mathrm{d} t\right) s^{j} & =\frac{\bar{B}(s)(\bar{u}(\mu))^{i}-\mu s^{i+1}}{\mu(\bar{B}(s)-\mu s)} \\
\int_{0}^{\infty} \mathrm{e}^{-\mu t} \bar{p}_{i 0}(t) \mathrm{d} t & =\mu^{-1}(\bar{u}(\mu))^{i}
\end{aligned}
$$

and

$$
\int_{0}^{\infty} \mathrm{e}^{-\mu t} \bar{p}_{i 0}^{\prime}(t) \mathrm{d} t=(\bar{u}(\mu))^{i}
$$

where $\bar{B}(s)=\sum_{j=0}^{\infty} \bar{b}_{j} s^{j}$ and $\bar{u}(\mu) \in(0,1)$ is the smallest positive root of $\bar{B}(s)-\mu s=0$.

Now, by substituting (3.4)-(3.5) and (3.7)-(3.8) into (3.6) and using some easy algebra, we obtain, for any $|s|<1$,

$$
\sum_{j=1}^{\infty}\left(\int_{0}^{\infty} \mathrm{e}^{-\mu t} \bar{p}_{i j}(t) \mathrm{d} t\right) s^{j}=\frac{s\left((\bar{u}(\mu))^{i}-s^{i}\right)}{\bar{B}(s)-\mu s}
$$

By noting that $\bar{B}(s)=s_{\lambda}^{-1} B\left(s_{\lambda} s\right)+\lambda s$ we obtain $\bar{B}^{\prime}(1)=B^{\prime}\left(s_{\lambda}\right)+\lambda$ and, thus, by the proof of Theorem 3.2, we know that $\bar{B}^{\prime}(1) \leq 0$, which in turn implies that $\lim _{\mu \downarrow} \bar{u}(\mu)=1$ (see properties (iv) and (v) of Lemma 3.1). Now letting $\mu \downarrow 0$ in (3.9), noting the just proven result that $\lim _{\mu \downarrow 0} \bar{u}(\mu)=1$, and using the fact that $\bar{B}(s)=s_{\lambda}^{-1} B\left(s_{\lambda} s\right)+\lambda s$ once again, immediately yields (3.2). Also (3.1) follows directly from (3.8). Hence, we have proved that (3.1) and (3.2) hold for any $\lambda \in\left(-\infty, \lambda_{C}\right]$.

Finally, we can rewrite (3.2) as

$$
\sum_{j=1}^{\infty}\left(\int_{0}^{\infty} \mathrm{e}^{\lambda t} p_{i j}(t) \mathrm{d} t\right) s_{\lambda}^{j-1} s^{j-1}=\frac{s_{\lambda}^{i}\left(1-s^{i}\right)}{B\left(s_{\lambda} s\right)+\lambda s_{\lambda} s}, \quad|s|<1 .
$$

Note that $\tilde{B}(s)=B\left(s_{\lambda} s\right)+\lambda s_{\lambda} s$ possesses all the properties of $B(s)$, specified in, for example, Lemma 2.1 of [7], and that we have both $\tilde{B}(1)=0$ and $\tilde{B}^{\prime}(1) \leq 0$; thus, by Lemma 2.2 of [7], the function $G_{\lambda}(s)=(1-s) / \tilde{B}(s)$ can be expanded as a Taylor series:

$$
G_{\lambda}(s)=\sum_{k=0}^{\infty} \tilde{g}_{k} s^{k}, \quad|s|<1,
$$

where $\tilde{g}_{k}=G_{\lambda}^{(k)}(0) / k$ !. Substituting the above expression into (3.10) and comparing the corresponding coefficients of $s^{j}$ immediately yields (3.3). The proof is complete.

\section{Invariant measures and quasistationary distributions}

We now turn our attention to the quasistationary distribution of the stopped $M^{X} / M / 1$ queue. We first consider the invariant measures. 
Theorem 4.1. Suppose that the generator matrix $\boldsymbol{Q}$ defined in (1.1)-(1.2) is conservative. Let $\left(p_{i j}(t) ; i, j \in \mathbb{Z}_{+}\right)$be the $Q$-function of the stopped $M^{X} / M / 1$ queueing process, and let $\lambda_{C}$ be the decay parameter for the $Q$-function on $C$. Then, for any $\lambda \in\left[0, \lambda_{C}\right]$, the following statements hold.

(i) There exists a $\lambda$-invariant measure $\left(m_{i} ; i \in C\right)$ for $\boldsymbol{Q}$ on $C$, which is unique up to constant multiples. Moreover, the generating function of this $\lambda$-invariant measure $M(s)=\sum_{i=1}^{\infty} m_{i} s^{i-1}$ takes the simple form

$$
M(s)=\frac{m_{1} b_{0}}{B(s)+\lambda s}, \quad|s|<s_{\lambda},
$$

where $s_{\lambda}$ is the smallest positive root of $B(s)+\lambda s=0$ and $m_{1}>0$ is a constant.

(ii) The measure $\left(m_{i} ; i \in C\right)$ is also a $\lambda$-invariant measure for $\left(p_{i j}(t) ; t \geq 0\right)$ on $C$.

(iii) The $\lambda$-invariant measure $\left(m_{i} ; i \in C\right)$ is convergent (i.e. $\left.\sum_{i \in C} m_{i}<\infty\right)$ if and only if $B^{\prime}(1)<0, \rho>1$ (including $\rho=+\infty$ ), and $0<\lambda \leq \lambda_{C}$, where $\rho$ is the convergence radius of $B(s)$.

Proof. For $\lambda \in\left[0, \lambda_{C}\right]$, let $s_{\lambda}$ denote the smallest positive root of $B(s)+\lambda s=0$. Consider

$$
\tilde{B}(s)=B\left(s_{\lambda} s\right)+\lambda s_{\lambda} s, \quad|s| \leq 1 .
$$

It is easy to see that $\tilde{B}(1)=0$ and $\tilde{B}^{\prime}(1) \leq 0$. By Lemma 2.3 of [7], $G(s)=(1-s) / \tilde{B}(s)$ is well defined at least in $(-1,1)$ and can be expanded as a Taylor series:

$$
G(s)=\sum_{k=0}^{\infty} g_{k} s^{k}, \quad|s|<1
$$

where the coefficients $g_{k}=G^{(k)}(0) / k !(k \geq 0)$ satisfy $0<g_{k} \leq g_{0}=1 / b_{0}(k \geq 1)$. Therefore,

$$
\frac{1}{\tilde{B}(s)}=\sum_{n=0}^{\infty}\left(\sum_{k=0}^{n} g_{k}\right) s^{n}, \quad|s|<1 .
$$

Choose $m_{1}>0$, and let

$$
m_{i+1}=m_{1} b_{0} s_{\lambda}^{-i} \sum_{k=0}^{i} g_{k}>0, \quad i \geq 1
$$

Then, for all $|s|<s_{\lambda}$,

$$
M(s)=\sum_{i=0}^{\infty} m_{i+1} s^{i}=m_{1} b_{0} \sum_{i=0}^{\infty}\left(\sum_{k=0}^{i} g_{k}\right)\left(\frac{s}{s_{\lambda}}\right)^{i}=\frac{m_{1} b_{0}}{\tilde{B}\left(s / s_{\lambda}\right)}=\frac{m_{1} b_{0}}{B(s)+\lambda s} .
$$

Hence,

$$
B(s) M(s)-m_{1} b_{0}=-\lambda M(s) s, \quad|s|<s_{\lambda},
$$

i.e.

$$
\sum_{j=1}^{\infty}\left(\sum_{i=1}^{j+1} m_{i} b_{j-i+1}\right) s^{j}=-\lambda M(s) s, \quad|s|<s_{\lambda}
$$


Therefore,

$$
\sum_{i=1}^{j+1} m_{i} b_{j-i+1}=-\lambda m_{j}, \quad j \geq 1,
$$

which shows that $\left(m_{i} ; i \in C\right)$ is a $\lambda$-invariant measure for $\boldsymbol{Q}$ on $C$.

To prove the uniqueness, let $s_{\lambda}$ denote the smallest positive root of $B(s)+\lambda s=0$ for any $\lambda \in\left[0, \lambda_{C}\right]$. By the above proof we see that the $\lambda$-invariant measure $\left(m_{i} ; i \in C\right)$ for $\boldsymbol{Q}$ on $C$, given in (4.2), satisfies (4.3), which can be further rewritten as

$$
m_{j+1}=\frac{-\lambda m_{j}-\sum_{i=1}^{j} m_{i} b_{j-i+1}}{b_{0}}, \quad j \geq 1 .
$$

Now, suppose that $\left(\tilde{m}_{i} ; i \in C\right)$ is another $\lambda$-invariant measure for $\boldsymbol{Q}$ on $C$, i.e.

$$
\sum_{i=1}^{j+1} \tilde{m}_{i} b_{j-i+1}=-\lambda \tilde{m}_{j}, \quad j \geq 1,
$$

or, equivalently,

$$
\tilde{m}_{j+1}=\frac{-\lambda \tilde{m}_{j}-\sum_{i=1}^{j} \tilde{m}_{i} b_{j-i+1}}{b_{0}}, \quad j \geq 1 .
$$

Let $c=\tilde{m}_{1} / m_{1}$. Then, by (4.4) and (4.5),

$$
\tilde{m}_{2}=\frac{-\lambda \tilde{m}_{1}-\tilde{m}_{1} b_{j-i+1}}{b_{0}}=\frac{-\lambda c m_{1}-c m_{1} b_{j-i+1}}{b_{0}}=c m_{2} .
$$

It follows from (4.4), (4.5), and mathematical induction, that $\tilde{m}_{j}=\mathrm{cm}_{j}$ for all $j \geq 1$. Therefore, the $\lambda$-invariant measure for $\boldsymbol{Q}$ on $C$ is unique up to constant multiples. Hence, (i) is proved.

Now, since our $q$-matrix $Q$ is bounded, then a positive measure $\left(m_{i} ; i \in C\right)$ is $\lambda$-invariant for $\boldsymbol{Q}$ on $C$ if and only if $\left(m_{i} ; i \in C\right)$ is $\lambda$-invariant for the minimal $\boldsymbol{Q}$-function $\boldsymbol{P}(t)$ on $C$. Hence, (ii) follows directly from (i).

Finally, note that $B(s)+\lambda s=0$ has a positive root $s_{*}>1$ if and only if $B^{\prime}(1)<0, \rho>1$, and $0<\lambda \leq \lambda_{C}$. Hence, (iii) follows from (4.1). The proof is complete.

Remark 4.1. Since a $\lambda$-invariant measure for $p_{i j}(t)$ on $C$ must be a $\lambda$-invariant measure for $Q$ on $C$, then Theorem 4.1 implies that the $\lambda$-invariant measure for $p_{i j}(t)$ on $C$ is unique up to constant multiples. Note that if $B^{\prime}(1)=0$ or if $B^{\prime}(1)<0$ with $\rho=1$ and thus $\lambda_{C}=0$, then in the statement of Theorem 4.1 and some other similar situations 'for any $\lambda \in\left[0, \lambda_{C}\right]$ ' should be read as ' $\lambda=0$ '.

Having given the $\lambda_{C}$-invariant measure on $C$. We now further consider the quasistationary distributions for $p_{i j}(t)$ on $C$.

Theorem 4.2. Suppose that the generator matrix $\boldsymbol{Q}$ defined in (1.1)-(1.2) is conservative. Let ( $p_{i j}(t) ; i, j \in \mathbb{Z}_{+}$) be the corresponding stopped $M^{X} / M / 1$ queueing process, and let $\lambda_{C}$ be the decay parameter for the $\boldsymbol{Q}$-function on $C$. Then there exists a quasistationary distribution for $p_{i j}(t)$ on $C$ if and only if $B^{\prime}(1)<0$ and $\rho>1$. Moreover, if these conditions hold then there 
exists a one-parameter family of quasistationary distributions $\left\{\left(m_{i}(\lambda) ; i \in C\right) ; \lambda \in\left(0, \lambda_{C}\right]\right\}$ which can be given by

$$
M_{\lambda}(s)=\frac{\lambda}{B(s)+\lambda s}, \quad|s|<s_{\lambda},
$$

where $M_{\lambda}(s)=\sum_{i=1}^{\infty} m_{i}(\lambda) s^{i-1}$ and $s_{\lambda}$ is the smallest positive root of $B(s)+\lambda s=0$.

Proof. By Proposition 3.1 of [28], a probability distribution $\left(m_{i} ; i \in C\right)$ on $C$ is a quasistationary distribution for $p_{i j}(t)$ on $C$ if and only if, for some $\lambda>0,\left(m_{i} ; i \in C\right)$ is $\lambda$-invariant for $p_{i j}(t)$ on $C$. Thus, the conclusions follow from Theorem 4.1.

Corollary 4.1 , below, shows that the $\lambda_{C}$-quasistationary distribution has some minimal properties among the family of quasistationary distributions specified in Theorem 4.2.

Corollary 4.1. Let $\left\{\left(m_{i}(\lambda) ; i \in C\right) ; \lambda \in\left(0, \lambda_{C}\right]\right\}$ be the one-parameter family of quasistationary distributions specified in Theorem 4.2 and let $X_{\lambda}\left(\lambda \in\left(0, \lambda_{C}\right]\right)$ be the corresponding random variable which obeys the distribution $\left(m_{i}(\lambda) ; i \in C\right)$. Then the $\lambda_{C}$-quasistationary distribution $\left(m_{i}\left(\lambda_{C}\right) ; i \in C\right)$ is the minimal one in the sense that its corresponding random variable $X_{\lambda_{C}}$ has the smallest mean value and the smallest variance. Moreover, for any $\lambda \in$ $\left(0, \lambda_{C}\right]$,

$$
\mathrm{E}\left[X_{\lambda}\right]=\sum_{i=1}^{\infty} i m_{i}(\lambda)=-\frac{B^{\prime}(1)}{\lambda}
$$

and

$$
\operatorname{var}\left(X_{\lambda}\right)=\sum_{i=1}^{\infty} m_{i}(\lambda)\left(i+\frac{B^{\prime}(1)}{\lambda}\right)^{2}=\frac{B^{\prime}(1)^{2}}{\lambda^{2}}-\frac{B^{\prime \prime}(1)-B^{\prime}(1)}{\lambda} .
$$

Proof. Both (4.7) and (4.8) are direct consequences of (4.6) and, thus, (4.7) attains its minimum value at $\lambda=\lambda_{C}$. Since $\lambda \leq \lambda_{C}$ and $\left(m_{i}(\lambda) ; i \in C\right)$ is a distribution and noting that the variance is positive, then by (4.8) we have

$$
\left(B^{\prime \prime}(1)-B^{\prime}(1)\right) \lambda_{C}<B^{\prime}(1)^{2} .
$$

Finally, it is easy to see that

$$
\frac{\partial}{\partial \lambda} \operatorname{var}\left(X_{\lambda}\right)<0 \quad \text { for all } \lambda \in\left(0, \lambda_{C}\right]
$$

and, therefore, $\operatorname{var}\left(X_{\lambda}\right)>\operatorname{var}\left(X_{\lambda_{C}}\right)$. The proof is complete.

\section{Examples}

In this section we present some examples to illustrate the results obtained in the previous sections.

Example 5.1. Let $\boldsymbol{Q}=\left(q_{i j} ; i, j \in \mathbb{Z}_{+}\right)$be a stopped $\mathrm{M} / \mathrm{M} / 1$ generator matrix defined as follows:

$$
q_{i j}= \begin{cases}b & \text { if } i \geq 1, j=i+1, \\ a & \text { if } i \geq 1, j=i-1, \\ -(a+b) & \text { if } i=j \geq 1 \\ 0 & \text { otherwise }\end{cases}
$$


where $a>0$ and $b>0$. The corresponding $\boldsymbol{Q}$-function is denoted by $\left(p_{i j}(t) ; i, j \in \mathbb{Z}_{+}\right)$. For this example, we have $\rho=+\infty$ and $B(s)=a-(a+b) s+b s^{2}$ and, thus, by Corollary 2.2, the decay parameter $\lambda_{C}$ of $C=\{1,2, \ldots\}$ and $s_{*}$ satisfy (2.13), i.e.

$$
\begin{gathered}
a-\left(a+b-\lambda_{C}\right) s_{*}+b s_{*}^{2}=0, \\
-(a+b)+2 b s_{*}=-\lambda_{C} .
\end{gathered}
$$

Solving (5.1) and (5.2) immediately yields

$$
\lambda_{C}=(\sqrt{a}-\sqrt{b})^{2}, \quad s_{*}=\sqrt{\frac{a}{b}} .
$$

For any $\lambda \in\left[0,(\sqrt{a}-\sqrt{b})^{2}\right]$, by Theorem 4.1, a $\lambda$-invariant measure $\left(m_{i}(\lambda) ; i \in C\right)$ for $\boldsymbol{Q}$ (or for $\left.p_{i j}(t)\right)$ on $C$ can be given by

$$
M_{\lambda}(s)=\frac{m_{1} a}{a-(a+b-\lambda) s+b s^{2}}, \quad|s|<\frac{a+b-\lambda-\sqrt{(a+b-\lambda)^{2}-4 a b}}{2 b},
$$

where $M_{\lambda}(s)=\sum_{i=1}^{\infty} m_{i}(\lambda) s^{i-1}$. In particular, a $\lambda_{C}$-invariant measure $\left(m_{i} ; i \in C\right)$ for $\boldsymbol{Q}$ (or for $\left.p_{i j}(t)\right)$ on $C$ is

$$
m_{1}>0, \quad m_{i}=i\left(\sqrt{\frac{b}{a}}\right)^{i-1} m_{1}, \quad i>1 .
$$

By Theorem 4.2, there exists a quasistationary distribution for $p_{i j}(t)$ on $C$ if and only if $a>b$. Under this condition, the one-parameter family of quasistationary distributions $\left\{\left(m_{i}(\lambda) ; i \in\right.\right.$ $\left.C) ; \lambda \in\left(0,(\sqrt{a}-\sqrt{b})^{2}\right]\right\}$ is given by (5.3) with $m_{1}=\lambda / a$. By Corollary 4.1, for any $\lambda \in\left(0,(\sqrt{a}-\sqrt{b})^{2}\right]$, we have

$$
\mathrm{E}\left[X_{\lambda}\right]=\sum_{i=1}^{\infty} i m_{i}(\lambda)=\frac{a-b}{\lambda} \downarrow \frac{a-b}{(\sqrt{a}-\sqrt{b})^{2}} \quad \text { as } \lambda \uparrow(\sqrt{a}-\sqrt{b})^{2}
$$

and

$$
\operatorname{var}\left(X_{\lambda}\right)=\frac{(a-b)^{2}-\lambda(a+b)}{\lambda^{2}} \downarrow \frac{2 \sqrt{a b}}{(\sqrt{a}-\sqrt{b})^{2}} \quad \text { as } \lambda \uparrow(\sqrt{a}-\sqrt{b})^{2} .
$$

In particular, one of the quasistationary distributions is

$$
m_{i}=i\left(1-\sqrt{\frac{b}{a}}\right)^{2}\left(\sqrt{\frac{b}{a}}\right)^{i-1}, \quad i>1 .
$$

Furthermore, for any $i, j \geq 1$, we have

$$
\int_{0}^{\infty} \exp \left(\lambda_{C} t\right) p_{i 0}^{\prime}(t) \mathrm{d} t=\left(\frac{a}{b}\right)^{i / 2}
$$

and

$$
\int_{0}^{\infty} \exp \left(\lambda_{C} t\right) p_{i j}(t) \mathrm{d} t=\frac{j \wedge i}{a}\left(\frac{b}{a}\right)^{(j-i-1) / 2} .
$$

The following example is a generalization of Example 5.1. 
Example 5.2. Let $\boldsymbol{Q}=\left(q_{i j} ; i, j \in \mathbb{Z}_{+}\right)$be a stopped $\mathrm{M}^{\mathrm{X}} / \mathrm{M} / 1$ generator matrix defined as follows:

$$
q_{i j}= \begin{cases}b & \text { if } i \geq 1, j=i+k, \\ a & \text { if } i \geq 1, j=i-1, \\ -(a+b) & \text { if } i=j \geq 1, \\ 0 & \text { otherwise, }\end{cases}
$$

where $a>0, b>0$, and $k \geq 1$. The corresponding $\boldsymbol{Q}$-function is denoted by $\left(p_{i j}(t) ; i, j \in\right.$ $\left.\mathbb{Z}_{+}\right)$. For this example, we still have $\rho=+\infty$ and $B(s)=a-(a+b) s+b s^{k+1}$; thus, by Corollary 2.2 , the decay parameter $\lambda_{C}$ of $C=\{1,2, \ldots\}$ and $s_{*}$ satisfy

$$
\begin{gathered}
a-\left(a+b-\lambda_{C}\right) s_{*}+b s_{*}^{k+1}=0, \\
-(a+b)+(k+1) b s_{*}=-\lambda_{C} .
\end{gathered}
$$

Solving (5.4) yields

$$
\lambda_{C}=a+b-(k+1) b\left(\frac{a}{k b}\right)^{k /(k+1)}, \quad s_{*}=\sqrt[k+1]{\frac{a}{k b}} .
$$

For any $\lambda \in\left[0, \lambda_{C}\right]$, by Theorem 4.1, a $\lambda$-invariant measure $\left(m_{i}(\lambda) ; i \in C\right)$ for $\boldsymbol{Q}$ (or for $\left.p_{i j}(t)\right)$ on $C$ can be expressed as

$$
M_{\lambda}(s)=\frac{m_{1} a}{a-(a+b-\lambda) s+b s^{k+1}}, \quad|s|<s_{\lambda},
$$

where $M_{\lambda}(s)=\sum_{i=1}^{\infty} m_{i}(\lambda) s^{i-1}$ and $s_{\lambda}$ is the smallest positive root of $a-(a+b-\lambda) s+b s^{k+1}=$ 0 . By Theorem 4.2, there exists a quasistationary distribution for $p_{i j}(t)$ on $C$ if and only if $a>k b$. Under this condition, the one-parameter family of quasistationary distributions $\left\{\left(m_{i}(\lambda) ; i \in C\right) ; \lambda \in\left(0, \lambda_{C}\right]\right\}$ is given by (5.5) with $m_{1}=\lambda / a$. Moreover, for any $\lambda \in$ $\left(0, a+b-(k+1) b(a / k b)^{k /(k+1)}\right]$, by Corollary 4.1 we have

$$
\mathrm{E}\left[X_{\lambda}\right]=\sum_{i=1}^{\infty} i m_{i}(\lambda)=\frac{a-k b}{\lambda} \downarrow \frac{a-k b}{a+b-(k+1) b(a / k b)^{k /(k+1)}} \quad \text { as } \lambda \uparrow \lambda_{C}
$$

and

$$
\operatorname{var}\left(X_{\lambda}\right)=\frac{(a-k b)^{2}-\lambda\left(a+k^{2} b\right)}{\lambda^{2}} .
$$

In Examples 5.1 and 5.2 the sequence $\left\{b_{j} ; j \geq 0\right\}$ is short tailed, i.e. there exist some $k \geq 2$ such that $b_{j}=0(j>k)$. Therefore, $B(s)$ is convergent on the whole real line $(-\infty,+\infty)$. Now we give another example in which the convergence radius of $B(s)$ is finite.

Example 5.3. Let $\boldsymbol{Q}=\left(q_{i j} ; i, j \in \mathbb{Z}_{+}\right)$be a stopped $\mathrm{M}^{\mathrm{X}} / \mathrm{M} / 1$ generator matrix defined as follows:

$$
q_{i j}= \begin{cases}\frac{b \theta^{j-i-1}}{(j-i+1)(j-i)} & \text { if } i \geq 1, j>i, \\ a & \text { if } i \geq 1, j=i-1, \\ -\left(a+b\left(\frac{1}{\theta}+\frac{1-\theta}{\theta^{2}} \ln (1-\theta)\right)\right) & \text { if } i=j \geq 1, \\ 0 & \text { otherwise, }\end{cases}
$$


where $a>0, b>0$, and $\theta \in(0,1]$. Note that if $\theta=1$ then, as a convention, we view $(1-\theta) \ln (1-\theta)=0$ in (5.6) and some similar expressions below. The corresponding $\boldsymbol{Q}$-function is denoted by $\left(p_{i j}(t) ; i, j \in \mathbb{Z}_{+}\right)$. It is easy to see that the convergence radius of $B(s)$ is $1 / \theta$ and, when $\theta \in(0,1)$,

$$
B(s)=a-\left(a+\frac{b(1-\theta)}{\theta^{2}} \ln (1-\theta)\right) s+\frac{b(1-\theta s) \ln (1-\theta s)}{\theta^{2}}, \quad|s| \leq \frac{1}{\theta} .
$$

For this example, there are two different situations. First, if $\theta<1$ then it is easy to see that

$$
B(s)-B^{\prime}(s) s=\frac{a \theta^{2}+b(\theta s+\ln (1-\theta s))}{\theta^{2}}=0
$$

has a unique root $s_{*} \in(0,1 / \theta)$. By Corollary 2.2, the decay parameter $\lambda_{C}$ of $C=\{1,2, \ldots\}$ is

$$
\lambda_{C}=-B^{\prime}\left(s_{*}\right)=a+\frac{b}{\theta}+b\left(\frac{\ln \left(1-\theta s_{*}\right)}{\theta}+\frac{(1-\theta) \ln (1-\theta)}{\theta^{2}}\right) .
$$

For any $\lambda \in\left[0, \lambda_{C}\right]$, by Theorem 4.1, the $\lambda$-invariant measure $\left(m_{i}(\lambda) ; i \in C\right)$ for $\boldsymbol{Q}$ (or for $\left.p_{i j}(t)\right)$ on $C$ can be given by

$$
M_{\lambda}(s)=\frac{m_{1} a}{B(s)+\lambda s}, \quad|s|<s_{\lambda},
$$

where $M_{\lambda}(s)=\sum_{i=1}^{\infty} m_{i}(\lambda) s^{i-1}$ and $s_{\lambda}$ is the smallest positive root of $B(s)+\lambda s=0$. By Theorem 4.2, there exists a quasistationary distribution for $p_{i j}(t)$ on $C$ if and only if

$$
a \theta^{2}+b \theta+b \ln (1-\theta)>0 .
$$

Under this condition, the one-parameter family of quasistationary distributions $\left\{\left(m_{i}(\lambda) ; i \in\right.\right.$ $\left.C) ; \lambda \in\left(0, \lambda_{C}\right]\right\}$ is given by (5.7) with $m_{1}=\lambda / a$.

Secondly, if $\theta=1$ then

$$
B(s)=a(1-s)+b(1-s) \ln (1-s), \quad|s| \leq 1,
$$

and $B^{\prime}(1)=+\infty$. Therefore,

$$
\lambda_{C}=a+b\left(1+\ln \left(1-s_{*}\right)\right)
$$

where $s_{*}$ is the unique root of $a+b(s+\ln (1-s))=0$ in $(0,1)$.

For any $\lambda \in\left[0, \lambda_{C}\right]$, by Theorem 4.1, a $\lambda$-invariant measure $\left(m_{i}(\lambda) ; i \in C\right)$ for $\boldsymbol{Q}$ (or for $\left.p_{i j}(t)\right)$ on $C$ can be given by

$$
M_{\lambda}(s)=\frac{m_{1} a}{B(s)+\lambda s}, \quad|s|<s_{\lambda},
$$

where $M_{\lambda}(s)=\sum_{i=1}^{\infty} m_{i}(\lambda) s^{i-1}$ and $s_{\lambda}$ is the smallest positive root of the equation

$$
a(1-s)+b(1-s) \ln (1-s)+\lambda s=0 .
$$

Finally, by Theorem 4.2, there does not exist any quasistationary distribution for $p_{i j}(t)$ on $C$. 
The following example provides an important and interesting case in which the decay parameter $\lambda_{C}$ cannot be obtained by finding the tangent line of $B(s)$.

Example 5.4. Suppose that $a>0$ and $\beta \in(0,1]$. Let

$$
b_{0}=a, \quad b_{1}=-a-h(\beta), \quad \text { and } \quad b_{k}=\frac{\beta^{k}}{(k-1) k(k+1)}, \quad k \geq 2,
$$

where $h(\beta)=\sum_{k=2}^{\infty} \beta^{k} /(k-1) k(k+1)$. Using this sequence, we may construct a matrix $\boldsymbol{Q}$ as in (1.1)-(1.2) and, hence, this $Q$ is the generator matrix of some stopped $M^{X} / M / 1$ queue. For this queueing model, we have

$$
B(s)=a-(a+h(\beta)) s+\sum_{k=2}^{\infty} \frac{\beta^{k} s^{k}}{(k-1) k(k+1)}, \quad s \in\left[0, \frac{1}{\beta}\right],
$$

and, thus, $\rho=1 / \beta<\infty$. The corresponding $Q$-function is denoted by $\left(p_{i j}(t) ; i, j \in \mathbb{Z}_{+}\right)$. It is easy to obtain

$$
B(s)-B^{\prime}(s) s=a-\sum_{k=2}^{\infty} \frac{\beta^{k} s^{k}}{k(k+1)} \downarrow a-\frac{1}{2} \quad \text { as } s \uparrow \frac{1}{\beta} .
$$

By Corollary 2.1 or Corollary 2.2, it can be seen that if $a \leq \frac{1}{2}$ then we may obtain the decay parameter by finding the tangent line as

$$
\lambda_{C}=-B^{\prime}\left(s_{*}\right)
$$

where $s_{*}$ is the unique root of $\sum_{k=2}^{\infty} \beta^{k} s^{k} / k(k+1)=a$. However, if $a>\frac{1}{2}$ then we have $B(\rho)-\rho B^{\prime}(\rho)>0$ and, thus, $\lambda_{C}$ cannot be obtained by finding the tangent line of $B(s)$. Notwithstanding this, by Corollary 2.1 we may still obtain

$$
\lambda_{C}=-B\left(\frac{1}{\beta}\right) \beta .
$$

Finally, for any $\lambda \in\left[0, \lambda_{C}\right]$, by Theorem 4.1, a $\lambda$-invariant measure $\left(m_{i}(\lambda) ; i \in C\right)$ for $\boldsymbol{Q}$ (or for $\left.p_{i j}(t)\right)$ on $C$ can be given by

$$
M_{\lambda}(s)=\frac{m_{1} a}{B(s)+\lambda s}, \quad|s|<s_{\lambda}
$$

where $M_{\lambda}(s)=\sum_{i=1}^{\infty} m_{i}(\lambda) s^{i-1}$ and $s_{\lambda}$ is the smallest positive root of $B(s)+\lambda s=0$. By Theorem 4.2, there exists a quasistationary distribution for $p_{i j}(t)$ on $C$ if and only if $\beta \in(0,1)$ and $B^{\prime}(1)<0$. Under this condition, the one-parameter family of quasistationary distributions $\left\{\left(m_{i}(\lambda) ; i \in C\right) ; \lambda \in\left(0, \lambda_{C}\right]\right\}$ is given by (5.8) with $m_{1}=\lambda / a$.

Note that this example also shows that even for the case in which $a>\frac{1}{2}$ we may still determine the sign of $B(\rho)-\rho B^{\prime}(\rho)$ and then apply Corollary 2.1 or Corollary 2.2 to obtain $\lambda_{C}$ without evaluating the completed value of $B(\rho)-\rho B^{\prime}(\rho)$. See Remark 2.3 for the comments made there. 


\section{Acknowledgements}

The authors would like to thank an anonymous referee who provided extremely helpful comments and suggestions, which led to a much improved presentation of the results. The work of Chen Anyue was substantially supported by a grant from the Research Grants Council of the Hong Kong Special Administrative Region, China, Project HKU 7010/06P. The work of Li Junping was supported by the National Natural Sciences Foundation of China (No.10771216).

\section{References}

[1] Anderson, W. (1991). Continuous-Time Markov Chains: An Applications-Oriented Approach. Springer, New York.

[2] Asmussen, S. (2003). Applied Probability and Queues, 2nd edn. Springer, New York.

[3] Bayer, N. And Boxma, O. J. (1996). Wiener-Hopf analysis of an M/G/1 queue with negative customers and of a related class of random walks. Queueing Systems 23, 301-316.

[4] Chaudhry, M. L. and Templeton, J. G. C. (1983). A First Course in Bulk Queues. John Wiley, New York.

[5] Chen, A. Y. and Renshaw, E. (1997). The M/M/1 queue with mass exodus and mass arrivals when empty. J. Appl. Prob. 34, 192-207.

[6] Chen, A. Y. and Renshaw, E. (2004). Markovian bulk-arriving queues with state-dependent control at idle time. Adv. Appl. Prob. 36, 499-524.

[7] Chen, A. Y., Li, J. P. and Ramesh, N. I. (2005). Uniqueness and extinction of weighted Markov branching processes. Method. Comput. Appl. Prob. 7, 489-516.

[8] Chen, M. F. (1992). From Markov Chains to Non-Equilibrium Particle Systems. World Scientific, Singapore.

[9] Chen, M. F. (2004). Eigenvalues, Inequalities, and Ergodic Theory. Springer, London.

[10] Chung, K. L. (1967). Markov Chains with Stationary Transition Probabilities, 2nd edn. Springer, New York.

[11] Daley, D. J. (1969). Quasi-stationary behaviour of a left-continuous random walk. Ann. Math. Statist. 40, 532-539.

[12] Darroch, J. N. and Seneta, E. (1967). On quasistationary distributions in absorbing continuous-time finite Markov chains. J. Appl. Prob. 4, 192-196.

[13] Dudin, A. AND Nishimura, S. (1999). A BMAP/SM/1 queueing system with Markovian arrival input of disasters. J. Appl. Prob. 36, 868-881.

[14] Flaspohler, D. C. (1974). Quasi-stationary distributions for absorbing continuous-time denumerable Markov chains. Ann. Inst. Statist. Math. 26, 351-356.

[15] Gelenbe, E. (1991). Product form networks with negative and positive customers. J. Appl. Prob. $28,656-663$.

[16] Gelenbe, E., Glynn, P. and Sigman, K. (1991). Queues with negative arrivals. J. Appl. Prob. 28, $245-250$.

[17] Gross, D. and Harris, C. M.(1985). Fundamentals of Queueing Theory. John Wiley, New York.

[18] Harrison, P. G. AND Pitel, E. (1993). Sojourn times in single-server queues with negative customers. J. Appl. Prob. 30, 943-963.

[19] Henderson, W. (1993). Queueing networks with negative customers and negative queue lengths. J. Appl. Prob. 30, 931-942.

[20] JaIn, G. And Sigman, K. (1996). A Pollaczek-Khintchine formula for M/G/1 queues with disasters. J. Appl. Prob. 33, 1191-1200.

[21] Kelly, F. P. (1983). Invariant measures and the generator. In Probability, Statistics, and Analysis (London Math. Soc. Lecture Notes Ser. 79), eds J. F. C. Kingman and G. E. Reuter, Cambridge University Press, pp. 143-160.

[22] KiJima, M. (1993). Quasi-limiting distributions of Markov chains that are skip-free to the left in continuous-time. J. Appl. Prob. 30, 509-517.

[23] Kingman, J. F. C. (1963). The exponential decay of Markov transition probability. Proc. London Math. Soc. 13, 337-358.

[24] Kleinrock, I. (1975). Queueing Systems, Vol. 1. John Wiley, New York.

[25] LuCAntoni, D. M. (1991). New results on the single server queue with a batch Markovian arrival process. Stoch. Models 7, 1-46.

[26] Lucantoni, D. M. and Neuts, M. F. (1994). Some steady-state distributions for the MAP/SM/1 queue. Stoch. Models 10, 575-589.

[27] Medhi, J.(1991). Stochastic Models in Queuing Theory. Academic Press, San Diego, CA.

[28] NaIR, M. G. AND Pollett, P. K. (1993). On the relationship between $\mu$-invariant measures and quasistationary distributions for continuous-time Markov chains. Adv. Appl. Prob. 25, 82-102.

[29] Neuts, M. F.(1979). A versatile Markovian point process. J. Appl. Prob. 16, 764-779.

[30] Neuts, M. F. (1981). Matrix-Geometric Solution in Stochastic Models: An Algorithmic Approach. Johns Hopkins University Press, Baltimore, MD. 
[31] Nishimura, S. And Sato, H. (1997). Eigenvalue expression for a batch Markovian arrival process. J. Operat. Res. Soc. Japan 40, 122-132.

[32] Norris, J. R. (1997). Markov Chains. Cambridge University Press.

[33] Parthasarathy, P. R. and Krishna Kumar, B. (1991). Density-dependent birth and death processes with state-dependent immigration. Math. Comput. Modelling 15, 11-16.

[34] Pollett, P. K. (1988). Reversibility, invariance and $\mu$-invariance. Adv. Appl. Prob. 20, 600-621.

[35] Pollett, P. K. (1995). The determination of quasi-instationary distribution directly from the transition rates of an absorbing Markov chain. Math. Comput. Modelling 22, 279-287.

[36] Pollett, P. K. (1999). Quasistationary distributions for continuous time Markov chains when absorption is not certain. J. Appl. Prob. 36, 268-272.

[37] Stadje, W. (1989). Some exact expressions for the bulk-arrival queue $\mathrm{M}^{\mathrm{X}} / \mathrm{M} / 1$. Queuing Systems 4, 85-92.

[38] Syski, R. (1992). Passage Times for Markov Chains. IOS Press, Amsterdam.

[39] Tweedie, R. L. (1974). Some ergodic properties of the Feller minimal process. Quart. J. Math. Oxford Ser. (2) 25, 485-495.

[40] VAn Doorn, E. A. (1985). Conditions for exponential ergodicity and bounds for the decay parameter of a birth-death process. Adv. Appl. Prob. 17, 514-530.

[41] VAN Doorn, E. A. (1991). Quasi-stationary distributions and convergence to quasi-stationarity of birth-death processes. Adv. Appl. Prob. 23, 683-700.

[42] Vere-Jones, D. (1962). Geometric ergidicity in denumerable Markov chains. Quart. J. Math. Oxford Ser. (2) 13, 7-28.

[43] Yaglom, A. M. (1947). Certain limit theorems of the theory of branching random processes. Dokl. Akad. Nauk $\operatorname{SSSR}(N$. S.) 56, 795-798. 